\title{
On Inverse Problems for Characteristic Sources in Helmholtz Equations
}

\author{
Carlos J. S. Alves, ${ }^{1}$ Roberto Mamud, ${ }^{2,3}$ Nuno F. M. Martins, ${ }^{4}$ and Nilson C. Roberty ${ }^{3}$ \\ ${ }^{1}$ CEMAT-IST, Instituto Superior Técnico, Lisboa, Portugal \\ ${ }^{2}$ Universidade Federal do Rio de Janeiro, Campus Macaé, Macaé, RJ, Brazil \\ ${ }^{3}$ Programa de Engenharia Nuclear, COPPE, Universidade Federal do Rio de Janeiro, Rio de Janeiro, RJ, Brazil \\ ${ }^{4}$ CEMAT-IST, Faculdade de Ciências e Tecnologia, Universidade Nova de Lisboa, Caparica, Portugal \\ Correspondence should be addressed to Roberto Mamud; rmamud@macae.ufrj.br
}

Received 8 December 2016; Accepted 24 January 2017; Published 27 February 2017

Academic Editor: Guido Ala

Copyright (C) 2017 Carlos J. S. Alves et al. This is an open access article distributed under the Creative Commons Attribution License, which permits unrestricted use, distribution, and reproduction in any medium, provided the original work is properly cited.

\begin{abstract}
We consider the inverse problem that consists in the determination of characteristic sources, in the modified and classical Helmholtz equations, based on external boundary measurements. We identify the location of the barycenter establishing a simple formula for symmetric shapes, which also holds for the determination of a single source point. We use this for the reconstruction of the characteristic source, based on the Method of Fundamental Solutions (MFS). The MFS is also applied as a solver for the direct problem, using an equivalent formulation as a jump or transmission problem. As a solver for the inverse problem, we may apply minimization using an equivalent reciprocity functional formulation. Numerical experiments with the barycenter and the boundary reconstructions are presented.
\end{abstract}

\section{Introduction}

In this paper we focus on the inverse problem that consists in the determination of characteristic sources. Inverse source problems have been addressed since 1938, in the context of gravimetry, with the seminal work of Novikov [1], where he was able to prove uniqueness in the two-dimensional Laplace equation model, for star-shaped characteristic sources. For more general geometries, it has been shown by Sakai [2] that there are connected domains for which the problem of reconstruction from the potential outside the source region presents no uniqueness.

Here we consider the inverse problem for characteristic sources, but in the case of Helmholtz equations. When the center is known, uniqueness was proven for a convex characteristic source (see Isakov [3]) with no boundary restrictions. However the proof of Novikov is no longer available by the lack of minimum-maximum principles, and uniqueness has not been established for star-shaped characteristic sources.

While considering Helmholtz equations in a bounded domain this will change the problem in two different ways.
If we consider the modified Helmholtz equation, useful for the transient heat problem, the associated boundary value problem has a unique solution, but the decay is exponential. This compromises the quality of the recovery in the inverse problem. On the other hand, considering the classical Helmholtz equation, the models wave phenomena such as acoustic waves, we must avoid values associated with the resonance frequencies, in which the direct problem has no unique solution.

In this paper we focus on two main issues related with the inverse characteristic source problem, that is, the determination of the barycenter of the characteristic source and the recovery of its geometry from a class of star-shaped characteristic sources. As a solver for the inverse problem, we may apply a minimization algorithm using an equivalent reciprocity functional formulation.

In general, there are restrictions on the number and type of sources that can be identified from boundary data. Several particular classes of problems have been considered: combinations of point sources-see [4-6]; linear/affine classes as in $[7,8]$; classes of characteristic sources (e.g., [9-16]); and in 
particular for the Helmholtz equation we refer to the papers $[17,18]$, where a full identification result was established, but using instead an interval of frequencies.

In this paper, we only consider boundary measurements given by a single frequency.

The paper is organized as follows. In Section 2, we present the direct and inverse problems for Helmholtz equations. In Section 2.1 the inverse jump problem is also presented and we show the equivalence between this problem and the inverse characteristic source problem, which motivates the adoption of the Method of Fundamental Solutions to solve the direct problem (see Section 2.2). The MFS has been applied to solve several inverse problems in recent years (see [19] for an account of this research).

In Section 3 we obtain a new formula for the determination of the barycenter for characteristic sources with symmetric star-shaped support and show that it is the same as obtained for the point source inverse problem. In Section 3.3 the boundary reconstruction is presented as a minimization problem, where the classical Levenberg-Marquardt method is used but the minimization is not considered in terms of the boundary data directly, but in terms of the reciprocity functional.

Numerical experiments related to the barycenter and boundary reconstruction are presented in Section 4. In simulations, we solve the direct problems for different numerical parameters and generate the synthetic experimental data, avoiding the inverse crimes. Beside this, a barycenter formula presented in (46) is tested in Section 4.1. Section 4.2 is entirely dedicated to numerical experiments related to the boundary reconstruction, for the modified and classical Helmholtz equations.

\section{The Helmholtz Inverse Source Problem}

Let $\Omega \subset \mathbb{R}^{N}$ be an open, simply connected and bounded set with $C^{1}$ boundary, $\partial \Omega$. Given the source term $f \in L^{2}(\Omega)$ and the Dirichlet data $g \in H^{1 / 2}(\partial \Omega)$, consider the following problem:

$$
\begin{aligned}
(-\Delta+\lambda) u=f, & \text { in } \Omega, \\
u=g, & \text { on } \partial \Omega,
\end{aligned}
$$

where we are considering the cases $\lambda=0$ (Laplace equation), $\lambda=\kappa^{2}>0$ (modified Helmholtz equation), and the usual Helmholtz equation $\lambda=-\kappa^{2}<0$, with $\kappa$ denoting the wave number.

Problem (1) has unique solution, except for some $\lambda<0$ (eigenvalues of the Dirichlet-Laplace operator for $\Omega$ ) [20], and we can define the direct problem as the problem of finding $\partial u / \partial v \in H^{-1 / 2}(\partial \Omega)$, with $u \in H^{1}(\Omega)$, from the source term $f$ and Dirichlet data $g$, where $v$ is the normal outward unity vector. On the other hand, the inverse source problem consists in, given the Cauchy data $\left\{g, g_{\nu}\right\} \in H^{1 / 2}(\partial \Omega) \times H^{-1 / 2}(\partial \Omega)$, finding the source term $f$.
For test functions $v \in \mathscr{H}_{\lambda}(\Omega):=\left\{v \in H^{1}(\Omega) ;(-\Delta+\lambda) v=\right.$ $0\}$, we introduce the reciprocity functional

$$
\mathscr{R}[f](v):=\int_{\partial \Omega} u \frac{\partial v}{\partial v}-v \frac{\partial u}{\partial v} d \sigma=\int_{\partial \Omega} g \frac{\partial v}{\partial v}-v g_{v} d \sigma,
$$

and using Green's second identity, we get

$$
\int_{\partial \Omega} g \frac{\partial v}{\partial v}-v g_{v} d \sigma=\int_{\Omega} v f d x
$$

for all $v \in \mathscr{H}_{\lambda}(\Omega)$. Therefore,

$$
\mathscr{R}[f](v)=\int_{\Omega} v f d x=\langle f, v\rangle_{L^{2}(\Omega)},
$$

with $v \in \mathscr{H}_{\lambda}(\Omega)$.

In next theorem we establish an equivalence between the source reconstruction from the Cauchy data and from the reciprocity functional.

Theorem 1. The Cauchy data uniquely determines the source $f$ if, and only if, $f$ is uniquely determined by $\mathscr{R}[f](v)$, for all $v \in \mathscr{H}_{\lambda}(\Omega)$.

Proof. Consider the sources $f_{1}$ and $f_{2}$ for problem (1). Then

$$
\begin{aligned}
\mathscr{R} & {\left[f_{1}\right](v)-\mathscr{R}\left[f_{2}\right](v) } \\
& =\int_{\partial \Omega}\left(u_{1}-u_{2}\right) \frac{\partial v}{\partial v}-v\left(\frac{\partial u_{1}}{\partial v}-\frac{\partial u_{2}}{\partial v}\right) d \sigma .
\end{aligned}
$$

Imposing the same Dirichlet data on the boundary,

$$
\mathscr{R}\left[f_{1}\right](v)-\mathscr{R}\left[f_{2}\right](v)=\int_{\partial \Omega}\left(g_{v}^{2}-g_{v}^{1}\right) v d \sigma,
$$

for all $v \in \mathscr{H}_{\lambda}(\Omega)$. Since $\mathscr{H}_{\lambda}(\Omega)$ is homeomorphic to $H^{1 / 2}(\partial \Omega)$ (e.g., [17]), we have

$$
\begin{aligned}
\mathscr{R} & {\left[f_{1}\right](v)-\mathscr{R}\left[f_{2}\right](v) } \\
& =\left\langle g_{v}^{2}-g_{v}^{1}, v\right\rangle_{H^{-1 / 2}(\partial \Omega) \times H^{1 / 2}(\partial \Omega)}, \quad \forall v \in H^{1 / 2}(\partial \Omega) .
\end{aligned}
$$

Therefore, $\mathscr{R}\left[f_{1}\right]=\mathscr{R}\left[f_{2}\right]$, in $\mathscr{H}_{\lambda}(\Omega)$, is equivalent to $g_{v}^{2}=$ $g_{v}^{1}$, in $H^{-1 / 2}(\partial \Omega)$.

2.1. Equivalence to an Inverse Jump Problem. We now show that the inverse source problem for characteristic source is equivalent to an inverse jump problem.

Consider problem (1) with source $f=h \chi_{\omega}$, where $h \neq 0$ is constant and $\omega \subset \Omega$ is an admissible source set. Note that we can rewrite this problem as the transmission problem

$$
\begin{aligned}
& (-\Delta+\lambda) u^{-}=h, \quad \text { in } \omega, \\
& (-\Delta+\lambda) u^{+}=0, \quad \text { in } \Omega \backslash \bar{\omega}, \\
& {[u]=0, \quad \text { on } \partial \omega \text {, }} \\
& {\left[\frac{\partial u}{\partial v}\right]=0, \quad \text { on } \partial \omega} \\
& u^{+}=g, \quad \text { on } \partial \Omega,
\end{aligned}
$$


where $[u]=u^{-}-u^{+}$denotes the jump between the inner and outer traces of the solution, respectively. Let $\phi$ be a particular solution of the equation

$$
(-\Delta+\lambda) \phi=h
$$

Notice that if $\lambda \neq 0$, we can take $\phi=h / \lambda$ and if $\lambda=0$, we can take $\phi(x)=h\|x\|^{2} / 4$. So, we can split (8) into two problems

$$
\begin{aligned}
(-\Delta+\lambda) u^{+} & =0, \quad \text { in } \Omega \backslash \bar{\omega}, \\
u^{+} & =u^{-}, \quad \text { on } \partial \omega, \\
\frac{\partial u^{+}}{\partial v} & =\frac{\partial u^{-}}{\partial v}, \quad \text { on } \partial \omega, \quad \text { on } \partial \Omega, \\
u^{+} & =g,
\end{aligned}
$$

and

$$
\begin{aligned}
(-\Delta+\lambda)\left(u^{-}-\phi\right) & =0, \quad \text { in } \omega, \\
u^{-}-\phi & =u^{+}-\phi, \quad \text { on } \partial \omega, \\
\frac{\partial}{\partial \nu}\left(u^{-}-\phi\right) & =\frac{\partial u^{+}}{\partial \nu}-\frac{\partial \phi}{\partial \nu}, \quad \text { on } \partial \omega .
\end{aligned}
$$

Thus, considering

$$
\vartheta= \begin{cases}u^{+}, & \text {in } \Omega \backslash \bar{\omega} \\ u^{-}-\phi, & \text { in } \omega\end{cases}
$$

we have that, up to $\phi$, problem (8) is equivalent to the inverse jump problem

$$
\begin{aligned}
(-\Delta+\lambda) \vartheta & =0, & \text { in } \Omega \backslash \partial \omega, \\
{[\vartheta] } & =-\phi, & \text { on } \partial \omega, \\
{\left[\frac{\partial \vartheta}{\partial \nu}\right] } & =-\frac{\partial \phi}{\partial \nu}, & \text { on } \partial \omega, \\
\vartheta & =g, & \text { on } \partial \Omega .
\end{aligned}
$$

Thus, denoting the reciprocity functional of this problem as $\mathscr{R}[\partial \omega](\cdot)$, we establish the following result.

Theorem 2. If $\Omega \backslash \bar{\omega}$ is connected, then $\mathscr{R}\left[\chi_{\omega}\right]=\mathscr{R}[\partial \omega]$. Therefore, the inverse source problem of characteristic source (1) is equivalent to inverse jump problem (13).

Proof. The reciprocity functional for the inverse jump problem, with $v \in \mathscr{H}_{\lambda}(\Omega)$, is given by, applying the Green formula to the connected set $\Omega \backslash \bar{\omega}$,

$$
\begin{aligned}
\mathscr{R}[\partial \omega](v)= & \int_{\partial \Omega}\left(\vartheta^{+} \frac{\partial v}{\partial \nu}-v \frac{\partial \vartheta^{+}}{\partial \nu}\right) d \sigma \\
= & -\int_{\partial \omega^{+}}\left(\vartheta^{+} \frac{\partial v}{\partial \nu}-v \frac{\partial \vartheta^{+}}{\partial \nu}\right) d \sigma \\
& +\int_{\Omega \backslash \bar{\omega}} \overbrace{\left(\vartheta^{+} \Delta v-v \Delta \vartheta^{+}\right)}^{=0} d \sigma .
\end{aligned}
$$

Notice that the orientation of the normal changes, by taking $\partial \omega^{-}$as the boundary of $\omega$, instead of $\partial \omega^{+}$as a part of the boundary of $\Omega \backslash \bar{\omega}$. Also, we have

$$
\begin{aligned}
& \int_{\partial \omega^{-}}\left(\vartheta^{-} \frac{\partial v}{\partial \nu}-\vartheta \frac{\partial u^{-}}{\partial \nu}\right) d \sigma=\int_{\omega} \overbrace{\left(\vartheta^{-} \Delta v-v \Delta \vartheta^{-}\right)}^{=0} d \sigma \\
& =0 .
\end{aligned}
$$

Then, for all $v \in \mathscr{H}_{\lambda}(\Omega)$,

$$
\begin{aligned}
\mathscr{R}[\partial \omega](v) & =\int_{\partial \omega^{+}}\left(\vartheta^{+} \frac{\partial v}{\partial \nu}-v \frac{\partial \vartheta^{+}}{\partial \nu}\right) d \sigma \\
& =-\int_{\partial \omega}\left([\vartheta] \frac{\partial v}{\partial v}-v\left[\frac{\partial \vartheta}{\partial \nu}\right]\right) d \sigma \\
& =\int_{\partial \omega}\left(\phi \frac{\partial v}{\partial v}-v \frac{\partial \phi}{\partial v}\right) d \sigma \\
& =\int_{\omega}(\phi \Delta v-v \Delta \phi) d x=\int_{\omega} f v d x \\
& =\mathscr{R}\left[\chi_{\omega}\right](v)
\end{aligned}
$$

using (2).

Remark 3. If $\Omega \backslash \bar{\omega}$ was not connected, this equivalence would not hold, in view of $\partial \omega^{+} \neq \partial \omega^{-}(=\partial \omega)$.

This jump or transmission problem will be solved using the Method of Fundamental Solutions (MFS) that we present next.

2.2. Solving the Direct Problem with MFS. The Method of Fundamental Solutions (MFS) is a meshless boundary method that has been widely studied in recent years. Its main goal is to approximate the solution of certain boundary value problems by a combination of fundamental solutions of the partial differential equation, in which the singularities lie outside the domain (e.g., [21]).

Furthermore, this method has become a popular tool because of its applicability in several areas where we can cite, as an example, $[7,8,19,22,23]$, including inverse problems.

Consider the direct problem

$$
\begin{aligned}
(-\Delta+\lambda) u=f, & \text { in } \Omega, \\
u=g, & \text { on } \partial \Omega,
\end{aligned}
$$

where the source term is $f=h \chi_{\omega}$, with $h \neq 0$ constant and $\omega \subset \Omega$ an open, connected, bounded subset with boundary $C^{1}, \partial \omega$.

Using Section 2.1, problem (17) can be rewritten as the transmission problem for $\phi(13)$, where

$$
\phi(x)= \begin{cases}\frac{h}{\lambda}, & \text { if } \lambda \neq 0, \\ h \frac{\|x\|^{2}}{4}, & \text { if } \lambda=0,\end{cases}
$$


and $\vartheta$ is given by (12). In this way, the functions $\vartheta^{+}$and $\vartheta^{-}$will be determined by the MFS. For this, consider the following expansions centered at the corresponding source points:

$$
\vartheta^{-}(x)=\sum_{i=1}^{p} \alpha_{i} \Phi_{\lambda}\left(\left|x-c_{i}\right|\right),
$$

with $c_{i} \in \gamma$, and

$$
\vartheta^{+}(x)=\sum_{j=1}^{q} \beta_{j}^{I} \Phi_{\lambda}\left(\left|x-a_{j}\right|\right)+\sum_{l=1}^{r} \beta_{l}^{E} \Phi_{\lambda}\left(\left|x-b_{l}\right|\right),
$$

with $a_{j} \in \Gamma^{I}$ and $b_{l} \in \Gamma^{E}$, where $\Phi_{\lambda}$ is a fundamental solution of Laplace or Helmholtz equation; that is,

$$
(-\Delta+\lambda) \Phi_{\lambda}=\delta,
$$

where $\delta$ is the Dirac distribution. Beside this, fictitious boundaries $\gamma, \Gamma^{I}$, and $\Gamma^{E}$ are chosen such that

(i) the curve $\gamma$ is outer of $\omega$, with $\gamma \subset \Omega \backslash \bar{\omega}$;

(ii) the curve $\Gamma^{I}$ is outer of $\Omega \backslash \bar{\omega}$, with $\Gamma^{I} \subset \omega$;

(iii) the curve $\Gamma^{E}$ is outer of $\Omega \backslash \bar{\omega}$, with $\Gamma^{E} \subset \Omega^{c}$.

So, 9 is a solution of (13) if it satisfies the following boundary conditions.

(i) $[\vartheta]=\vartheta^{-}-\vartheta^{+}=-\phi$, on $\partial \omega$; that is, for $x \in \partial \omega$,

$$
\begin{gathered}
\sum_{i=1}^{p} \alpha_{i} \Phi_{\lambda}\left(\left|x-c_{i}\right|\right)-\sum_{j=1}^{q} \beta_{j}^{I} \Phi_{\lambda}\left(\left|x-a_{j}\right|\right) \\
-\sum_{l=1}^{r} \beta_{l}^{E} \Phi_{\lambda}\left(\left|x-b_{l}\right|\right)=-\phi(x) .
\end{gathered}
$$

In general, it is considered that $2 m+n \geq 2(p+q+r)$ and the system should be considered in the least squares sense. In the $2 \mathrm{D}$ case, the fundamental solution $\Phi_{\lambda}$ can be taken as

$$
\Phi_{\lambda}(x)= \begin{cases}\frac{1}{2 \pi} K_{0}(\sqrt{\lambda}|x|), & \text { if } \lambda>0, \\ -\frac{1}{2 \pi} \log (|x|), & \text { if } \lambda=0, \\ \frac{i}{4} H_{0}^{(1)}(\sqrt{-\lambda}|x|), & \text { if } \lambda<0,\end{cases}
$$

where $H_{0}^{(1)}=J_{0}+i Y_{0}$ is a Hänkel function and $J_{0}, K_{0}, Y_{0}$ are Bessel functions. (ii) $[\partial \vartheta / \partial \nu]=\partial \vartheta^{-} / \partial \nu-\partial \vartheta^{+} / \partial \nu=-\partial \phi / \partial \nu$, on $\partial \omega$; that is,

$$
\begin{gathered}
\sum_{i=1}^{p} \alpha_{i} \frac{\partial}{\partial \nu}\left[\Phi_{\lambda}\left(\left|x-c_{i}\right|\right)\right]-\sum_{j=1}^{q} \beta_{j}^{I} \frac{\partial}{\partial \nu}\left[\Phi_{\lambda}\left(\left|x-a_{j}\right|\right)\right] \\
-\sum_{l=1}^{r} \beta_{l}^{E} \frac{\partial}{\partial \nu}\left[\Phi_{\lambda}\left(\left|x-b_{l}\right|\right)\right]=-\frac{\partial \phi}{\partial \nu}(x),
\end{gathered}
$$

$x \in \partial \omega$.

(iii) $\vartheta=g$, on $\partial \Omega$; that is,

$$
\sum_{j=1}^{q} \beta_{j}^{I} \Phi_{\lambda}\left(\left|x-a_{j}\right|\right)+\sum_{l=1}^{r} \beta_{l}^{E} \Phi_{\lambda}\left(\left|x-b_{l}\right|\right)=g(x),
$$

$x \in \partial \Omega$.

In this way, taking collocation points $x_{m^{*}} \in \partial \omega$, with $m^{*}=$ $1,2, \ldots, m$, and $x_{n^{*}} \in \partial \Omega$, with $n^{*}=1,2, \ldots, n$, we can write the above problem as a linear system:

$$
\mathrm{M} \alpha=b,
$$

where

$$
\begin{gathered}
\alpha=\left[\begin{array}{l}
{\left[\alpha_{i}\right]_{p \times 1}} \\
{\left[\beta_{j}^{I}\right]_{q \times 1}} \\
{\left[\beta_{l}^{E}\right]_{r \times 1}}
\end{array}\right]_{(p+q+r) \times 1}, \\
b=\left[\begin{array}{c}
{\left[-\phi\left(x_{m^{*}}\right)\right]_{m \times 1}} \\
{\left[-\frac{\partial \phi}{\partial \nu}\left(x_{m^{*}}\right)\right]_{m \times 1}} \\
{\left[g\left(x_{n^{*}}\right)\right]_{n \times 1}}
\end{array}\right]_{(2 m+n) \times 1}
\end{gathered}
$$

and the matrix $\mathrm{M},(2 m+n) \times(p+q+r)$, of the linear system coefficients, is given by

$$
\left[\begin{array}{ccc}
{\left[\Phi_{\lambda}\left(\left|x_{m^{*}}-c_{i}\right|\right)\right]_{m \times p}} & {\left[-\Phi_{\lambda}\left(\left|x_{m^{*}}-a_{j}\right|\right)\right]_{m \times q}} & {\left[-\Phi_{\lambda}\left(\left|x_{m^{*}}-b_{l}\right|\right)\right]_{m \times r}} \\
{\left[\frac{\partial \Phi_{\lambda}}{\partial \nu}\left(\left|x_{m^{*}}-c_{i}\right|\right)\right]_{m \times p}} & {\left[-\frac{\partial \Phi_{\lambda}}{\partial \nu}\left[\left(\left|x_{m^{*}}-a_{j}\right|\right)\right]\right]_{m \times q}} & {\left[-\frac{\partial \Phi_{\lambda}}{\partial \nu}\left[\left(\left|x_{m^{*}}-b_{l}\right|\right)\right]\right]_{m \times r}} \\
{[0]_{n \times p}} & {\left[\Phi_{\lambda}\left(\left|x_{n^{*}}-a_{j}\right|\right)\right]_{n \times q}} & {\left[\Phi_{\lambda}\left(\left|x_{n^{*}}-b_{l}\right|\right)\right]_{n \times r}}
\end{array}\right] .
$$

Therefore, solving the linear system, in the least squares sense, by Tikhonov regularization

$$
\left(M^{\top} M+\tau\right) \alpha=M^{\top} b
$$

( $\tau \approx 0$ being a regularization parameter), we find a solution, $\vartheta$, of (13), in which we may generate an approximation of the direct solution

$$
\frac{\partial \vartheta}{\partial \nu}, \quad \text { on } \partial \Omega
$$


This approximation will be used to generate the synthetic data in Section 3.3.

Remark 4. In the numerical experiments of Section 4, the fictitious boundaries $\gamma, \Gamma^{I}$, and $\Gamma^{E}$ were chosen as circles each one in the correspondent region. The choice of the location of source points is not unique and its best location is a research subject (see, e.g., [24-27]).

\section{Barycenter and Point Source Determination}

We consider the special class of admissible sets.

Definition 5. A subset $\omega \subset \mathbb{R}^{N}$ is said to be star-shaped with respect to the origin, if there exists a function $R: \mathbb{S}^{N-1} \rightarrow \mathbb{R}_{*}^{+}$, such that

$$
\omega=\left\{\rho \theta \in \mathbb{R}^{N} ; 0<\rho<R(\theta), \theta \in \mathbb{S}^{N-1}\right\},
$$

where $R \in C^{1}\left(\mathbb{S}^{N-1}\right)$ is the positive radius function.

We say that a subset $\omega \subset \mathbb{R}^{N}$ is a symmetric star-shaped set if it is star-shaped and the function $R$ satisfies

$$
R(\theta)=R(-\theta), \quad \forall \theta \in \mathbb{S}^{N-1} .
$$

When the barycenter $p$ is not at the origin, we can use the notation $\omega+p$ to refer to a shifted domain.

In this section we establish a result for the determination of the barycenter, in the case of symmetric star-shaped characteristic sources for the Helmholtz equation, with no restriction on the sign of $\lambda$, and we bound the reciprocity functional.

In Section 3.2 we see that the same formula also holds for the determination of a point source.

3.1. Determination of the Barycenter. In the case $\lambda=0$ (Laplace equation), since $v=1$ and $v(x)=x_{j}(j=1, \ldots, N)$ are functions of $\mathscr{H}_{0}(\Omega)$, it is well known that the barycenter is determined from

$$
p_{j}=\frac{\mathscr{R}\left[\chi_{\omega}\right]\left(x_{j}\right)}{\mathscr{R}\left[\chi_{\omega}\right](1)}, \quad j=1, \ldots, N,
$$

where $\mathscr{R}\left[\chi_{\omega}\right](1)=\int_{\omega} h(x) d x$ and $\mathscr{R}\left[\chi_{\omega}\right]\left(x_{j}\right)=\int_{\omega} h(x) x_{j} d x$ (for any weight function $h>0$ ).

Consider now $\kappa=\sqrt{\lambda} \neq 0$, and $f=\chi_{\omega}$ (therefore $\left.h=1\right)$.

Using a test function $v_{\varphi}(x)=e^{\kappa \varphi \cdot(x-p)}$, where $\varphi \in \mathbb{S}^{N-1}$ and $p \in \mathbb{R}^{N}$ are arbitrary, the reciprocity functional is

$$
\begin{aligned}
\mathscr{R} & {\left[\chi_{\omega}\right]\left(e^{\kappa \varphi \cdot(x-p)}\right) } \\
& =\int_{\partial \Omega} g \kappa(\varphi \cdot \nu) e^{\kappa \varphi \cdot(x-p)}-g_{\nu} e^{\kappa \varphi \cdot(x-p)} d \sigma .
\end{aligned}
$$

On the other hand, by (3), we have, denoting $\mathscr{R}\left[\chi_{\omega}\right](\varphi):=$ $\mathscr{R}\left[\chi_{\omega}\right]\left(e^{\kappa \varphi \cdot x}\right)$,

$$
\begin{aligned}
e^{-\kappa \varphi \cdot p} \mathscr{R}\left[\chi_{\omega}\right](\varphi) & =\mathscr{R}\left[\chi_{\omega}\right]\left(e^{\kappa \varphi \cdot(x-p)}\right) \\
& =\int_{\omega} e^{\kappa \varphi \cdot(x-p)} d x .
\end{aligned}
$$

In the following, we will suppose that the origin of the coordinate system, $p$, is the barycenter of an admissible source set $\omega \subset \Omega$, in which we suppose symmetric star-shaped set, whose boundary, $\partial \omega$, is parametrized by a function $R$.

Therefore, by (35),

$$
\begin{aligned}
e^{-\kappa \varphi \cdot p} \mathscr{R}\left[\chi_{\omega}\right](\varphi) & =\int_{\omega} e^{\kappa \varphi \cdot(x-p)} d x \\
& =\int_{\mathbb{S}^{N-1}} \int_{0}^{R(\theta)} e^{\kappa \rho \varphi \cdot \theta} \rho^{N-1} d \rho d \theta \\
& =: F[R](\varphi)
\end{aligned}
$$

for all $\varphi \in \mathbb{S}^{N-1}$.

Proposition 6. Let $\omega \subset \Omega$ be a symmetrical star-shaped subset, whose boundary, $\partial \omega$, is parametrized by the function $R \in L^{1}\left(\mathbb{S}^{N-1}\right)$. Then

$$
F[R](\varphi)=F[R](-\varphi), \quad \forall \varphi \in \mathbb{S}^{N-1}
$$

Proof. In fact, let $\varphi \in \mathbb{S}^{N-1}$ be an arbitrary direction. Consider the sets

$$
\begin{aligned}
& \mathbb{S}_{ \pm}^{\varphi}:=\left\{\theta \in \mathbb{S}^{N-1} ; \pm \varphi \cdot \theta>0\right\}, \\
& \mathbb{S}_{0}^{\varphi}:=\left\{\theta \in \mathbb{S}^{N-1} ; \varphi \cdot \theta=0\right\} .
\end{aligned}
$$

So, $\mathbb{S}^{N-1}=\mathbb{S}_{-}^{\varphi} \cup \mathbb{S}_{0}^{\varphi} \cup \mathbb{S}_{+}^{\varphi}$ and then

$$
\begin{aligned}
F[R](\varphi)= & \int_{\mathbb{S}_{+}^{\varphi}} \int_{0}^{R(\theta)} e^{\kappa \rho \varphi \cdot \theta} \rho^{N-1} d \rho d \theta \\
& +\int_{\mathbb{S}_{0}^{\varphi}} \int_{0}^{R(\theta)} \rho^{N-1} d \rho d \theta \\
& +\int_{\mathbb{S}_{-}^{\varphi}} \int_{0}^{R(\theta)} e^{\kappa \rho \varphi \cdot \theta} \rho^{N-1} d \rho d \theta .
\end{aligned}
$$

On the other hand, note that

$$
\begin{aligned}
F[R](-\varphi)= & \int_{\mathbb{S}_{+}^{-\varphi}} \int_{0}^{R(\theta)} e^{-\kappa \rho \varphi \cdot \theta} \rho^{N-1} d \rho d \theta \\
& +\int_{\mathbb{S}_{0}^{-\varphi}} \int_{0}^{R(\theta)} \rho^{N-1} d \rho d \theta \\
& +\int_{\mathbb{S}_{-}^{-\varphi}} \int_{0}^{R(\theta)} e^{-\kappa \rho \varphi \cdot \theta} \rho^{N-1} d \rho d \theta .
\end{aligned}
$$


Therefore, since $\mathbb{S}_{ \pm}^{-\varphi}=\mathbb{S}_{\mp}^{\varphi}, \mathbb{S}_{0}^{-\varphi}=\mathbb{S}_{0}^{\varphi}$, and the star-shaped set is symmetrical, then

$$
\begin{aligned}
& \int_{\mathbb{S}_{+}^{-\varphi}} \int_{0}^{R(\theta)} e^{-\kappa \rho \varphi \cdot \theta} \rho^{N-1} d \rho d \theta \\
& =\int_{\mathbb{S}_{-}^{\varphi}} \int_{0}^{R(\theta)} e^{\kappa \rho \varphi \cdot \theta} \rho^{N-1} d \rho d \theta, \\
& \int_{\mathbb{S}_{-}^{-\varphi}} \int_{0}^{R(\theta)} e^{-\kappa \rho \varphi \cdot \theta} \rho^{N-1} d \rho d \theta \\
& =\int_{\mathbb{S}_{+}^{\varphi}} \int_{0}^{R(\theta)} e^{\kappa \rho \varphi \cdot \theta} \rho^{N-1} d \rho d \theta
\end{aligned}
$$

and we obtain

$$
F[R](\varphi)=F[R](-\varphi) .
$$

In this way, since $\varphi$ is arbitrary, we have $F[R](\varphi)=F[R](-\varphi)$, for all $\varphi \in \mathbb{S}^{N-1}$.

So, by proposition 6 and by (35),

$$
e^{-\kappa \varphi \cdot p} \mathscr{R}\left[\chi_{\omega}\right](\varphi)=e^{\kappa \varphi \cdot p} \mathscr{R}\left[\chi_{\omega}\right](-\varphi) .
$$

Therefore,

$$
e^{2 \kappa \varphi \cdot p}=\frac{\mathscr{R}\left[\chi_{\omega}\right](\varphi)}{\mathscr{R}\left[\chi_{\omega}\right](-\varphi)}
$$

that is,

$$
\varphi \cdot p=\frac{1}{2 \kappa} \ln \left(\frac{\mathscr{R}\left[\chi_{\omega}\right](\varphi)}{\mathscr{R}\left[\chi_{\omega}\right](-\varphi)}\right) .
$$

Remark 7. Note that in this case we have used the boundary parametrization associated with the symmetric star-shaped characteristic sources in the proof.

Thereby, we establish the following new result for the barycenter identification of symmetrical star-shaped supports in the inverse source problem posed on the modified Helmholtz operator.

Theorem 8. Let $\omega \subset \Omega$ be a symmetrical star-shaped subset and let $f(x)=\chi_{\omega}(x)$ be the source term for the inverse problem. Then the barycenter, $p$, of $\omega$ is uniquely determined by

$$
\varphi \cdot p=\frac{1}{2 \kappa} \ln \left(\frac{\mathscr{R}\left[\chi_{\omega}\right](\varphi)}{\mathscr{R}\left[\chi_{\omega}\right](-\varphi)}\right),
$$

where $\varphi \in \mathbb{S}^{N-1}$.

3.2. Determination of Point Sources. In this subsection we will study the inverse source problem for a single point source. Consider the inverse problem with a single point source of type

$$
f_{p}(x)=\alpha \delta_{p}(x),
$$

where $\delta_{p}(x)$ is the Dirac delta distribution centered at the point $p \in \mathbb{R}^{N}$ and $\alpha>0$ is the intensity of this source. In this way, taking $v_{\varphi}(x)=e^{\kappa \varphi \cdot x}$ in (4), with $\varphi \in \mathbb{S}^{N-1}$,

$$
\mathscr{R}\left[f_{p}\right](\varphi):=\mathscr{R}\left[f_{p}\right]\left(v_{\varphi}\right)=\alpha e^{\kappa \varphi \cdot p}, \quad \forall \varphi \in \mathbb{S}^{N-1} .
$$

Thus, since $\mathscr{R}\left[f_{p}\right](-\varphi)=\alpha e^{-\kappa \varphi \cdot p}$, the source intensity is determined in the following way:

$$
\alpha^{2}=\mathscr{R}\left[f_{p}\right](\varphi) \cdot \mathscr{R}\left[f_{p}\right](-\varphi) .
$$

On the other hand, the source position is obtained from

$$
\varphi \cdot p=\frac{1}{2 \kappa} \ln \left(\frac{\mathscr{R}\left[f_{p}\right](\varphi)}{\mathscr{R}\left[f_{p}\right](-\varphi)}\right),
$$

where $\varphi \in \mathbb{S}^{N-1}$. The coordinates of the point source are simply given by $p_{k}=\mathbf{e}_{k} \cdot p$, with $\varphi=\mathbf{e}_{k}$ being the canonical basis vector.

Remark 9. Note that, by (50) and (46), we obtain the same expression for the barycenter of symmetric star-shaped sources and for the position of a single point source.

3.3. Determination of the Source Support Boundary from Cauchy Data. Consider the subsets $\omega, \widetilde{\omega} \subset \mathbb{R}^{2}$, with same barycenter, whose boundaries, $\partial \omega$ and $\partial \widetilde{\omega}$, are parametrized, respectively, by $R(t)$ and by a truncated Fourier series

$$
\widetilde{R}(t)=\alpha_{0}+\sum_{n=1}^{(\mathrm{NP}-1) / 2}\left(\alpha_{n} \cos \left(\frac{n t}{2}\right)+\alpha_{n+1} \sin \left(\frac{n t}{2}\right)\right),
$$

with $t \in[0,2 \pi]$. Here, we suppose that the barycenter of $\widetilde{\omega}$ is the origin.

Remark 10. In an arbitrary coordinate system, where the barycenter of $\widetilde{\omega}$ is not the origin, the boundary points are given by

$$
\begin{aligned}
& \left(x_{c}, y_{c}\right) \\
& +\left(\alpha_{0}+\sum_{n=1}^{(\mathrm{NP}-1) / 2} \alpha_{n} \cos \left(\frac{n t}{2}\right)+\alpha_{n+1} \sin \left(\frac{n t}{2}\right)\right) \\
& \cdot(\cos (t), \sin (t)),
\end{aligned}
$$

with $t \in[0,2 \pi]$. The barycenter position $p=\left(x_{c}, y_{c}\right)$ will be calculated using the reconstruction formula (46); see Section 3.1.

Remark 11 . In $\mathbb{R}^{N}, \partial \widetilde{\omega}$ is parametrized, in the barycenter coordinate system, by truncated $N$-dimensional spherical harmonics series of the radius $\widetilde{R}(\theta) \in L^{1}\left(\mathbf{S}^{N-1}\right)$, which express the distance between the centroid and the correspondent source boundary point.

Using results from Section 3.1, we can assume that the sources have the same barycenter. Theorem 2 shows us how 
to implement the boundary reconstruction algorithm using solutions of direct problem (1) with $f=h \chi_{\omega}$ or equivalently solving interface problem (13) with traces at the source boundary of equivalent particular solution.

Remark 12. The normal trace of trial solutions with parameters guess is calculated solving equivalent interface problem (13) with the corresponding trials. As we have pointed out before, the Method of Fundamental Solution is appropriated for the Helmholtz equation model.

Related to these subsets $\omega$ and $\widetilde{\omega}$, we have the associated reciprocity functionals $\mathscr{R}\left[\chi_{\omega}\right](v)$ and $\mathscr{R}\left[\chi_{\widetilde{\omega}}\right](v)$, for $v \in$ $\mathscr{H}_{\lambda}(\Omega)$.

Considering, in $\mathbb{R}^{2}$, test functions $\nu_{\tau}=e^{\kappa(\cos \tau, \sin \tau) \cdot x}$ and taking $M$ different directions $\tau_{i}=i(2 \pi / M), i=1, \ldots, M$, we define the following discrete functional to be minimized:

$$
J\left(v_{\tau_{i}}\right):=\sum_{i=1}^{M}\left(\mathscr{R}\left[\chi_{\omega}\right]\left(v_{\tau_{i}}\right)-\mathscr{R}\left[\chi_{\widetilde{\omega}}\right]\left(v_{\tau_{i}}\right)\right)^{2} .
$$

Discrete functional (53) represents a possible misfit between the reciprocity functional related to the sources $\chi_{\omega}$ and $\chi_{\widetilde{\omega}}$. Furthermore, note that

(i) the given $\mathscr{R}\left[\chi_{\omega}\right]\left(v_{\varphi}\right)$ depends only on Cauchy data;

(ii) the guessed $\mathscr{R}\left[\chi_{\tilde{\omega}}\right]\left(v_{\varphi}\right)$ can be calculated by solving Laplace-Helmholtz direct problem (17) with a trial source term guessed for parameters $\alpha_{n}$.

We present a numerical optimization method to approximate the boundary support which is based on a LevenbergMarquardt algorithm implementation for the nonlinear least squares formulation given by (53).

Remark 13. In $\mathbb{R}^{N}$ problems, we can define the functional to be minimized as

$$
J\left(v_{\theta}\right):=\int_{\mathbb{S}^{N-1}}\left(\mathscr{R}\left[\chi_{\omega(\alpha)}\right]\left(v_{\theta}\right)-\mathscr{R}\left[\chi_{\widetilde{\omega}}\right]\left(v_{\theta}\right)\right)^{2} d \theta,
$$

valid for test functions $v_{\theta}(x)=e^{\kappa \theta \cdot x} \in \mathscr{H}_{\lambda}(\Omega)$, with $|\theta|=1$.

In the next sections we present some bidimensional numerical experiments for the minimization of functional (53), defined above, using the Levenberg-Marquardt algorithm.

\section{Numerical Experiments}

When we study inverse source problems with characteristic sources defined by star-shaped sets, there are two pieces of information that we aim to retrieve: the barycenter of the source support and the boundary parametrization.

4.1. Numerical Experiments for Barycenter Determination. The barycenter reconstruction problem was studied in Section 3.1, and the main objective is to verify the accuracy of the new formula proposed in Theorem 8.
TABLE 1: Barycenter error: circular case.

\begin{tabular}{lcc}
\hline & Barycenter calculated & Position error \\
\hline Noise 0\% & $(-0.3,-0.2)$ & $3.06719 \times 10^{-9}$ \\
Noise 1\% & $(-0.309532,-0.190297)$ & 0.0136012 \\
Noise 5\% & $(-0.324528,-0.144536)$ & 0.0606459 \\
Noise 10\% & $(-0.368817,-0.135365)$ & 0.0944107 \\
\hline
\end{tabular}

TABLE 2: Barycenter error: circular case $-1 \%$ fixed Dirichlet noise.

\begin{tabular}{lcc}
\hline & Barycenter calculated & Position error \\
\hline Noise 0\% & $(-0.296432,-0.209535)$ & 0.0101805 \\
Noise 1\% & $(-0.316809,-0.183237)$ & 0.0237395 \\
Noise 5\% & $(-0.332893,-0.181616)$ & 0.0376822 \\
Noise 10\% & $(-0.343313,-0.181448)$ & 0.0471187 \\
\hline
\end{tabular}

TABLE 3: Barycenter error: circular case-10\% fixed Dirichlet noise.

\begin{tabular}{lcc}
\hline & Barycenter calculated & Position error \\
\hline Noise 0\% & $(-0.238834,-0.206423)$ & 0.0615019 \\
Noise 1\% & $(-0.33233,-0.126357)$ & 0.0804273 \\
Noise 5\% & $(-0.335185,-0.118567)$ & 0.0887092 \\
Noise 10\% & $(-0.264065,-0.0816816)$ & 0.123655 \\
\hline
\end{tabular}

In the numerical Examples 1, 2; and 3 we considered $\lambda=$ 1. In Example $4, \lambda=-1$ was considered.

Beside this, relative noise over the collocation points of domain was imposed; that is, the boundary $\partial \Omega$ was discretized and the reciprocity functional was determined with the Dirichlet data, in each element of this point list. This value was multiplied by $\left(1+\zeta_{1}\right)$, where $\zeta_{1} \in(-\varepsilon, \varepsilon)$ was given by a random function with uniform probability distribution.

Example 1. In this bidimensional experiment we consider circular geometries, with $\kappa=1$. In this case $\Omega=B_{1}(0,0)$ and $\omega=B_{0.3}(-0.3,-0.2)$, using the notation $B_{\rho}(c)$, where $\rho$ stands for the radius and $c$ for the center.

The direct problem was solved using the Method of Fundamental Solutions (MFS), where the projection of a solution on the boundary $\partial \Omega$ was taken as Dirichlet data, considering 629 collocation points and 252 point sources in the MFS. For the determination of the barycenter, the reciprocity functional was calculated considering the Cauchy data from the solution of the direct problem, $u$. The Dirichlet and Neumann data were given by the trace $\gamma_{0} u$ and the normal trace $\gamma_{1} u$, on $\partial \Omega$.

Thus, the influence of relative noise of $1 \%, 5 \%$, and $10 \%$ in the Cauchy data was studied, and the results are shown in Table 1. In this case, it was considered that Dirichlet and Neumann data have the same noise level.

In Table 2, $1 \%$ noise in the Dirichlet data was fixed, and the noise in the Neumann data ranges up to $10 \%$. On the other hand, in Table 3, 10\% noise in the Dirichlet data was fixed and the noise in the Neumann data ranges up to $10 \%$. The results were worse than when $1 \%$ noise was considered in the Dirichlet data. 
TABLE 4: Barycenter error: 7-star-shaped case.

\begin{tabular}{lcc}
\hline & Barycenter calculated & Position error \\
\hline Noise 0\% & $(0.300056,-0.0999748)$ & $6.13069 \times 10^{-5}$ \\
Noise $1 \%$ & $(0.300221,-0.100107)$ & $2.45237 \times 10^{-4}$ \\
Noise 5\% & $(0.298224,-0.100325)$ & $1.80583 \times 10^{-3}$ \\
Noise 10\% & $(0.297488,-0.101851)$ & $3.12016 \times 10^{-3}$ \\
\hline
\end{tabular}

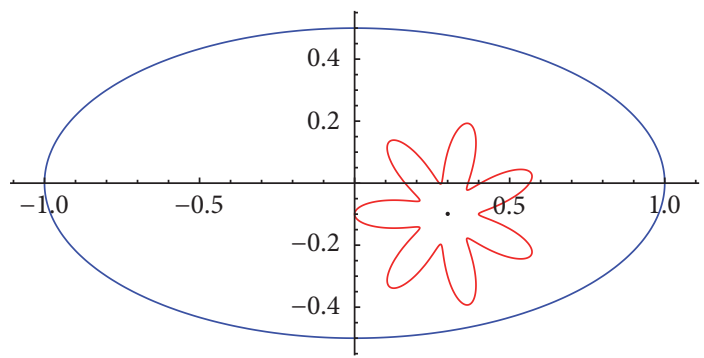

FIgURE 1: Problem domain: 7-star-shaped inside ellipse case.

Example 2. In this experiment we considered the domain $\Omega$ as the interior of the ellipse parametrized by $R(t)=$ $(\cos (t), 0.5 \sin (t)), t \in[0,2 \pi]$, and the support of the source as the star-shaped set $\omega$, with barycenter $(0.3,-0.1)$, and boundary parametrized by $r(t)=(0.3+(0.2-$ $0.1 \cos (7 t)) \cos (t),-0.1+(0.2-0.1 \cos (7 t)) \sin (t)), t \in[0,2 \pi]$, as shown in Figure 1.

In this problem, we consider null Dirichlet data and the direct problem was solved by the MFS (again with 629 collocation points and 252 sources).

The Dirichlet and Neumann data were perturbed with the same amount of relative noise. The results are given in Table 4 .

Example 3. In this experiment, the domain $\Omega$ was considered as the unitary circle, centered at the origin, and the support of the source $\omega$ as the interior of the square, centered at $\left(x_{c}, y_{c}\right)=$ $(0.4,-0.2)$, with parametrization

$$
r(t)= \begin{cases}\frac{0.3}{\cos (t)}, & \text { if }-\frac{\pi}{4} \leq t<\frac{\pi}{4}, \\ \frac{0.3}{\sin (t)}, & \text { if } \frac{\pi}{4} \leq t<\frac{3 \pi}{4}, \\ \frac{-0.3}{\cos (t)}, & \text { if } \frac{3 \pi}{4} \leq t<\frac{5 \pi}{4}, \\ \frac{-0.3}{\sin (t)}, & \text { if } \frac{5 \pi}{4} \leq t<\frac{7 \pi}{4},\end{cases}
$$

as shown in Figure 2.

We again considered null Dirichlet data in the direct problem and solved it by the MFS with only 200 collocation points and 100 source points, and the results are shown in Table 5.

Example 4. In this experiment, the domain $\Omega$ was considered as the interior of unitary circle centered at the origin and the
TABLE 5: Barycenter error: square case.

\begin{tabular}{lcc}
\hline & Barycenter calculated & Position error \\
\hline Noise 0\% & $(0.400002,-0.199989)$ & $1.15749 \times 10^{-5}$ \\
Noise 1\% & $(0.399996,-0.199778)$ & $2.21949 \times 10^{-4}$ \\
Noise 5\% & $(0.401539,-0.201175)$ & $1.9363 \times 10^{-3}$ \\
Noise 10\% & $(0.397216,-0.196766)$ & $4.26754 \times 10^{-3}$ \\
\hline
\end{tabular}

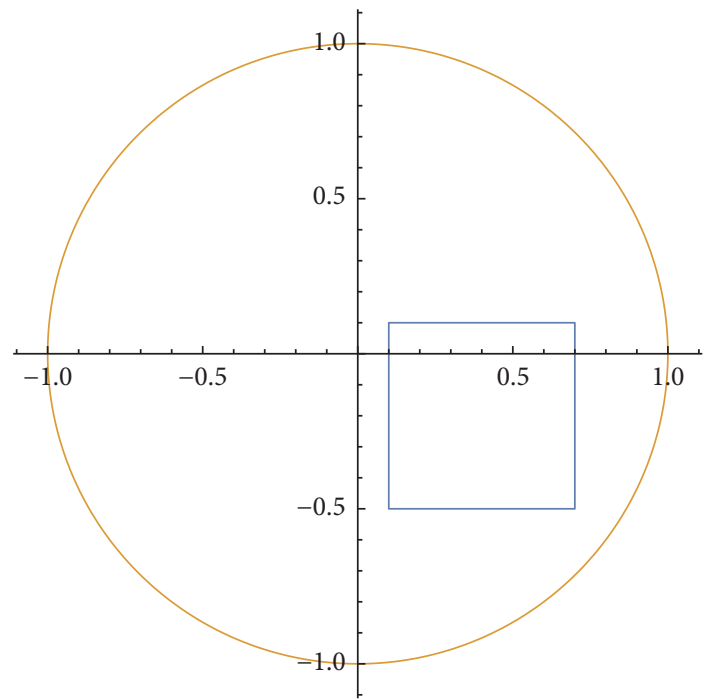

Figure 2: Problem domain: square inside circle case.

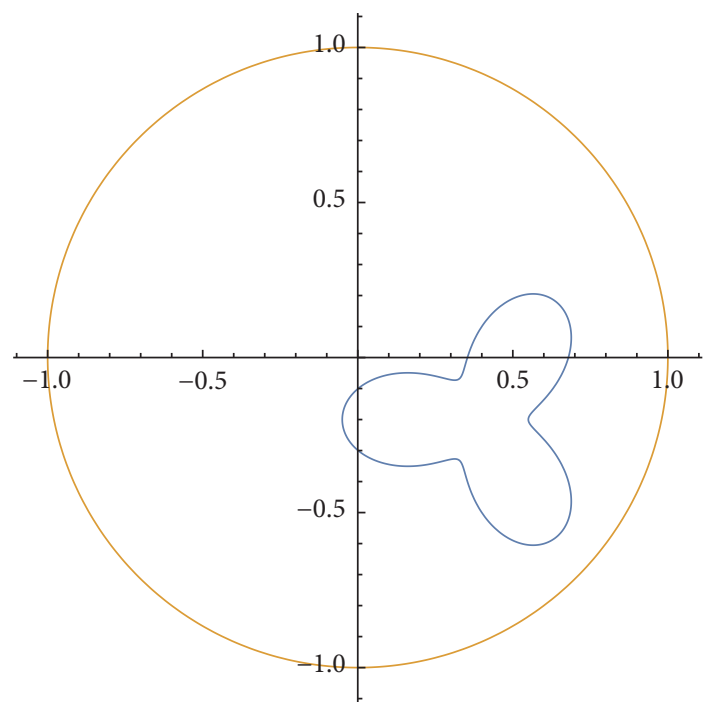

Figure 3: Problem domain: 3-star-shaped inside circle case, $\kappa$ complex.

support of source $\omega$ as the star-shaped set, with barycenter $\left(x_{c}, y_{c}\right)=(0.4,-0.2)$, whose boundary is parametrized by $r(t)=0.3-0.15 \cos (3 t)$, as shown in Figure 3 .

The main difference between this experiment and the others is the fact that here we consider $\lambda=-1$; that is, $\kappa=\sqrt{\lambda}=i$, 
TABLE 6: Barycenter error: case $\lambda<0$.

\begin{tabular}{lcr}
\hline & Barycenter calculated & Position error \\
\hline Noise 0\% & $\left(0.400697+9.46942 \times 10^{-7} i,-0.2+6.43473 \times 10^{-8} i\right)$ & $6.96922 \times 10^{-4}$ \\
Noise 1\% & $\left(0.400715+9.52632 \times 10^{-7} i,-0.199901+1.10503 \times 10^{-8} i\right)$ & $7.22001 \times 10^{-4}$ \\
Noise 5\% & $\left(0.398665+7.72773 \times 10^{-7} i,-0.201086-3.34906 \times 10^{-8} i\right)$ & $1.72124 \times 10^{-3}$ \\
Noise 10\% & $\left(0.396216+9.64432 \times 10^{-7} i,-0.198987-4.3447 \times 10^{-7} i\right)$ & $3.91735 \times 10^{-3}$ \\
\hline
\end{tabular}

a complex number. Consequently, the fundamental solution considered in the MFS method is given by

$$
\Phi_{\kappa}(x)=\frac{i}{4} H_{0}^{(1)}(\sqrt{-\lambda}|x|),
$$

where $H_{0}^{(1)}$ is the Hänkel function of first kind of zero order, and the results include neglectible complex numbers. As in the last experiment, we consider 200 collocation points and 100 source points, with null Dirichlet data in direct problem. Again the obtained results were good, as shown in Table 6.

All these experiments show that the formula proposed for determination of centroid proved to be very efficient, even with considerable amounts of noise level.

4.2. Numerical Experiments for Boundary Reconstruction. In this section we study the boundary reconstruction problem for inverse characteristic source problem. The boundary support reconstruction has been studied by many authors through several numerical methods to estimate this unknown parametrization. In this work, we use the Method of Fundamental Solution (MFS) to estimate the solution of direct problem and the Levenberg-Marquardt algorithm to minimize the functional (53) and estimate the support $\omega$.

4.2.1. Initial Guess for the Parameters. Another feature that is possible to obtain is the effective or mean radius, $r_{M}=\alpha_{0}$, the first term in the Fourier series parametrization. A guess for this parameter can be obtained by changing the test function for modified Helmholtz problem by the test function for the Laplace problem identically equal 1 (when $\kappa$ is small). In this way,

$$
\begin{gathered}
v \simeq 1 \Longrightarrow \\
\mathscr{R}\left[\chi_{\omega}\right](v) \simeq \mathscr{R}_{L}\left[f_{p}\right](1),
\end{gathered}
$$

where $\mathscr{R}\left[\chi_{\omega}\right](v)$ and $\mathscr{R}_{L}\left[f_{p}\right](1)$ are the reciprocity functional for modified Helmholtz inverse characteristic source problem, with test function $v$, and for Laplace inverse point source problem, with test function identically equal 1, respectively. So,

$$
\begin{aligned}
\mathscr{R}\left[\chi_{\omega}\right](v) & \simeq-\int_{\omega} 1 d x=-\mu(\omega) \simeq-\alpha \\
& =\mathscr{R}_{L}\left[f_{p}\right](1),
\end{aligned}
$$

where $\mu(\omega)$ is the Lebesgue measure of the subset $\omega \subset \Omega$. In this way, we obtain the following approximation for the bidimensional case

$$
\begin{aligned}
& \mu(\omega) \approx \pi r_{M}^{2} \simeq \alpha \Longrightarrow \\
& r_{M} \simeq \sqrt{\frac{\alpha}{\pi}}
\end{aligned}
$$

The initial guess for the other parameters are taken as zero; that is, we adopted $\alpha_{0}=\left(r_{M}, 0, \ldots, 0\right)$, as starting parameter value in the iterative minimization algorithm of least squares functional (53) done using the LevenbergMarquardt method. The algorithm has been checked for different numbers of parameters NP with good performance. In these experiments we adopted NP $=7$.

4.2.2. Kinds of Noise Considered. In the following experiments, two kinds of noise were considered: the relative and the absolute.

The relative and absolute noise are imposed over the measurements of the discretized boundary of domain, that is, on the MFS collocation points. In this way, the Cauchy data, in each element of this list, is multiplied by random functions with uniform probability distribution, $\zeta_{1} \in\left(-\varepsilon_{1}, \varepsilon_{1}\right)$, and $\zeta_{2} \in\left(-\varepsilon_{2}, \varepsilon_{2}\right)$. If $g$ is the measured data on the boundary, then

$$
\widetilde{g}=g\left(1+\zeta_{1}\right)+\zeta_{2}
$$

In Section 4.2.3 we implemented two experiments considering $\lambda>0$. In experiment 5 , was considered the influence of relative noise, but with a $\kappa$ variation, domain variation, and support variation. In experiment 6 , the square was considered as domain, without the influence of noise. In Section 4.2.4, $\lambda<0$, that is, $\kappa$ complex, and the action of both kinds of noise were considered.

4.2.3. Numerical Experiments for Boundary Reconstruction: Case $\lambda>0$. In the next experiments, $\lambda=1$ was considered and the damping factor, in Levenberg-Marquardt algorithm, was considered initially equal to 0.001 and, for each iteration, it is multiplied by a decay factor 0.1 . The initial values of damping factor and the decay factor were obtained empirically, after several experiments. Beside this, in all experiments $M=36$ directions were taken, to minimize functional (53).

Furthermore, all experiments were implemented in Mathematica software, version 9 , in a notebook with processor 


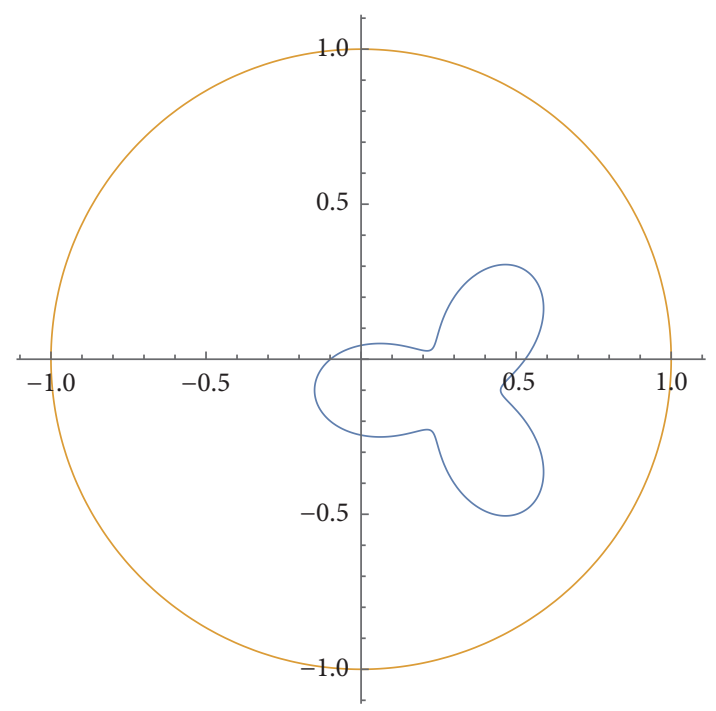

(a) Domain considered

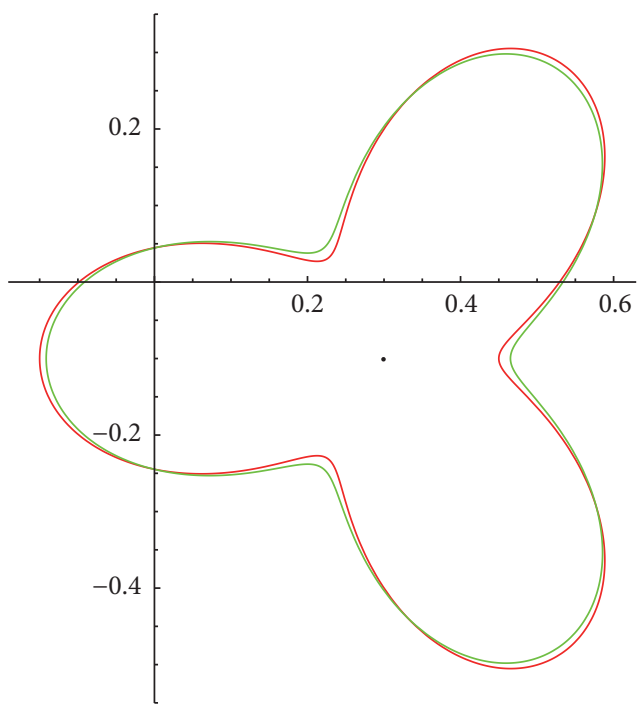

(b) Reconstruction, after 7 iterations, no noise

FIgURE 4: Case $\lambda>0$. Experiment with domain of radius 1 .

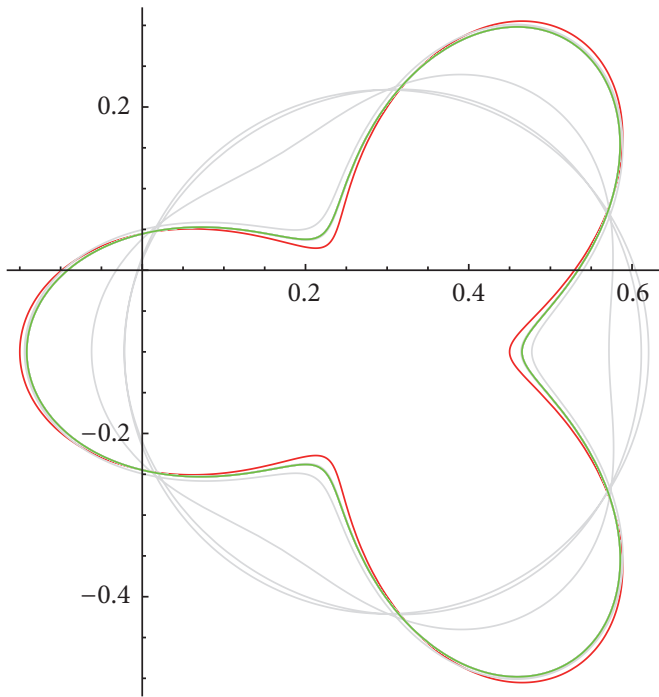

(a) $1 \%$

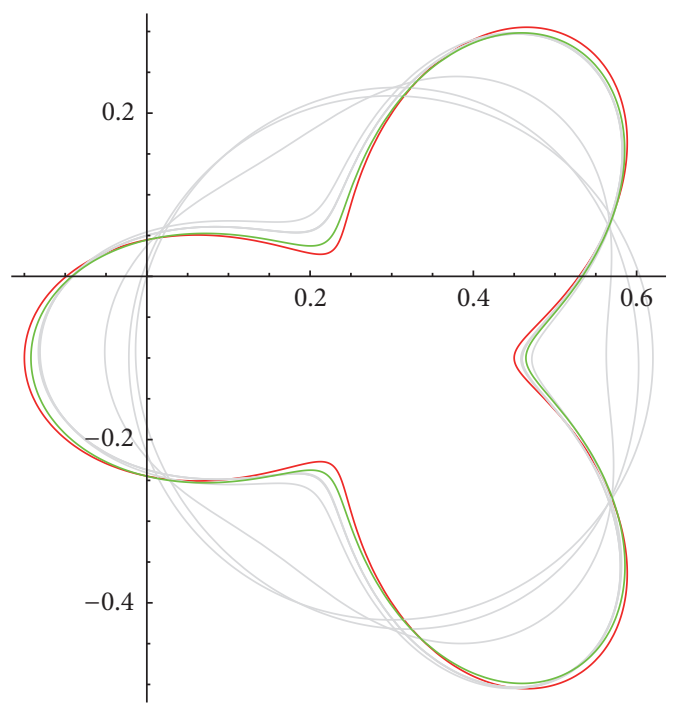

(c) $10 \%$

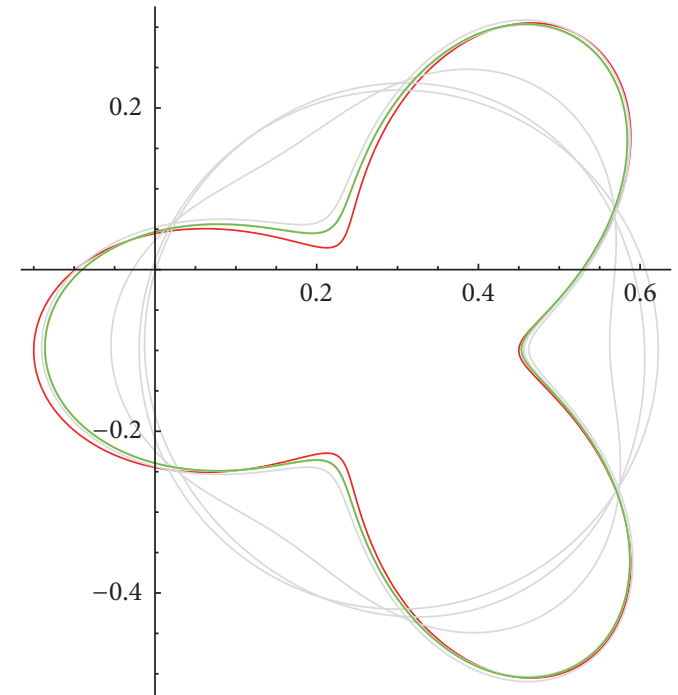

(b) $5 \%$

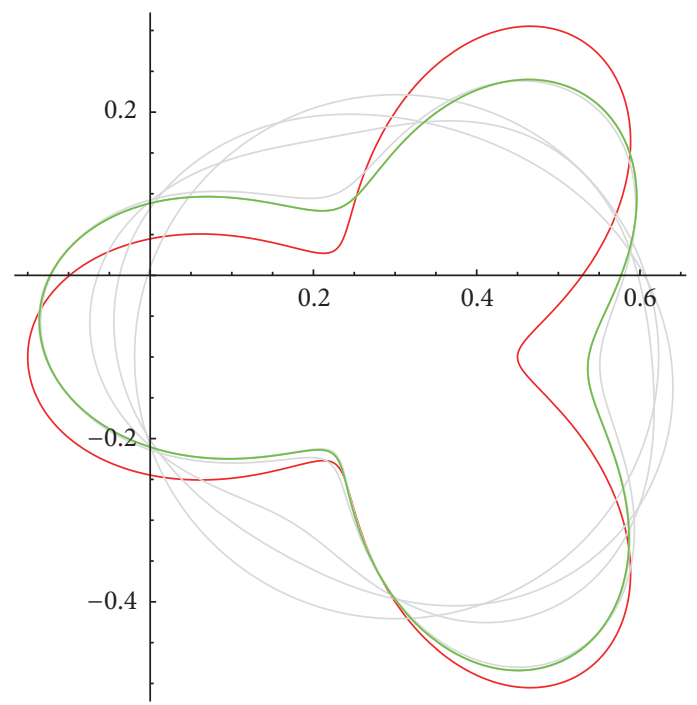

(d) $30 \%$

Figure 5: Case $\lambda>0$. Relative Noise. Domain with radius 1. 


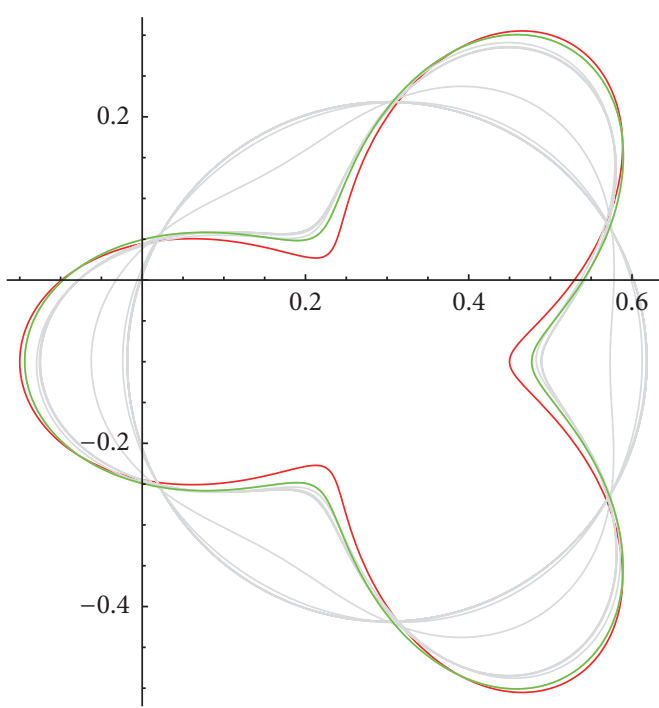

(a) $\kappa=0.1$

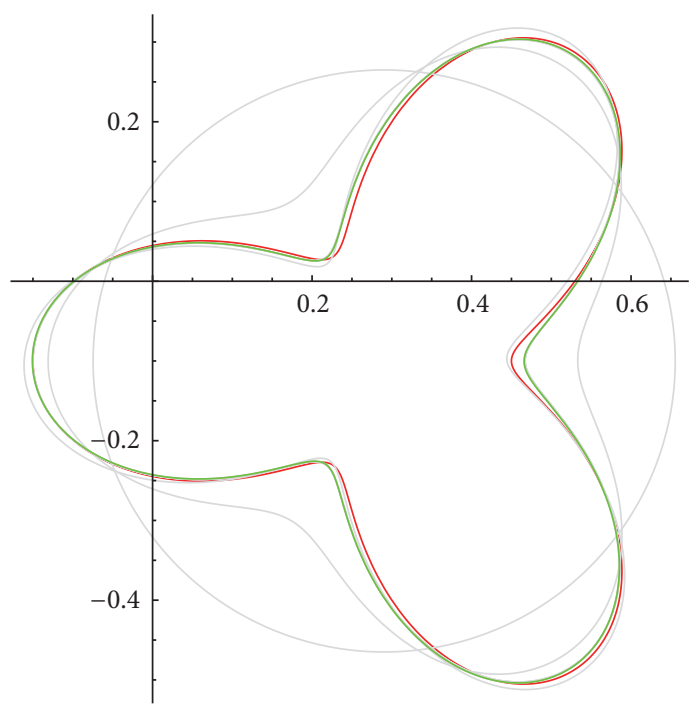

(c) $\kappa=4$

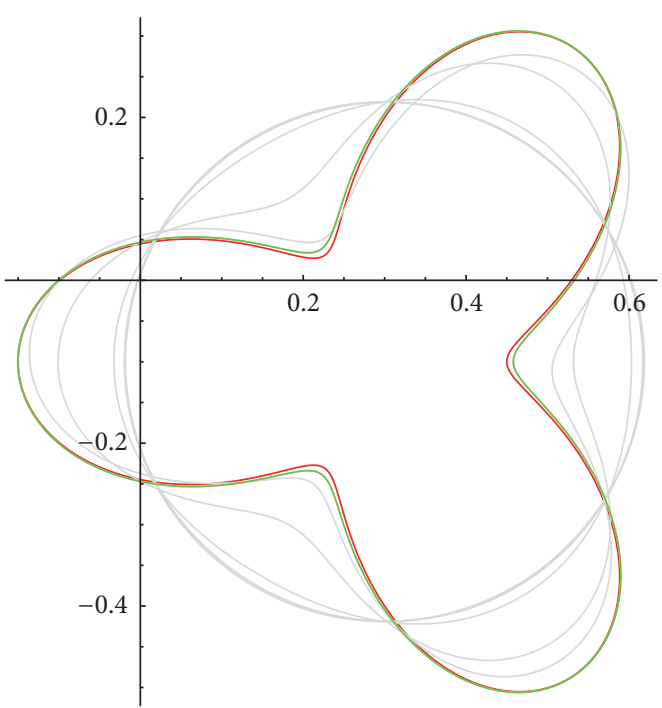

(b) $\kappa=0.55$

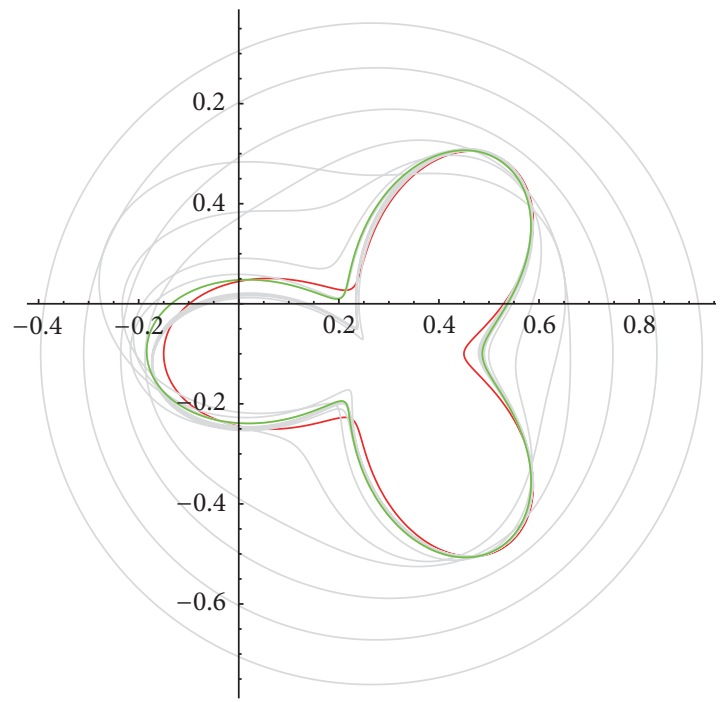

(d) $\kappa=10$

FIgURE 6: Case $\lambda>0 . \kappa$ variation. Domain with radius 1.

Intel Core i7, 2.8 GHz, RAM memory 4 GB, $1333 \mathrm{MHz}$ DDR3 with operational system OS X 10.9.5.

Example 5. In this experiment, $\Omega$ as a unitary circle and a support $\omega$ were considered, with center in $\left(x_{c}, y_{c}\right)=$ $(0.3,-0.1)$, whose boundary, $\partial \omega$, is parametrized by $r(t)=$ $1.1-0.6 \cos (3 t)$, as shown in Figure 4(a), where the Dirichlet data was considered null.

Beside this, the number of parameters in Fourier expansion was considered as 7 ; that is, $\mathrm{NP}=7$. In the original problem the MFS was considered with 200 collocation points and 100 source points, and for the MFS reconstruction 160 collocation points and 80 source points were considered. The initial parameter vector in reconstruction was $\left(r_{M}, 0,0,0,0,0,0\right)$, with mean radius $r_{M}=0.321$, calculated by (59).
After 7 iterations (of approximately 25 seconds each), the reconstruction was obtained without noise shown in Figure 4(b), where the figure in red is the original and in green is the reconstructed one.

Beside this, relative noise of $1 \%, 5 \%$, and $10 \%$ was also considered in the Neumann data. In all cases good reconstructions were obtained in the fifth iteration, as shown in Figure 5 (again, the figure in red is the original support, in green is the best reconstruction, and in grey is the reconstructions for each minimization iteration).

Another interesting feature is the influence of $\kappa$. In experiments related to Figure $5, \kappa=1$ was considered. In Figure 6 we present the reconstructions with $\kappa=0.1, \kappa=$ $0.55, \kappa=4$, and $\kappa=10$, and as the value of $\kappa$ increases, the value of mean radius also increases, as it can be seen in 


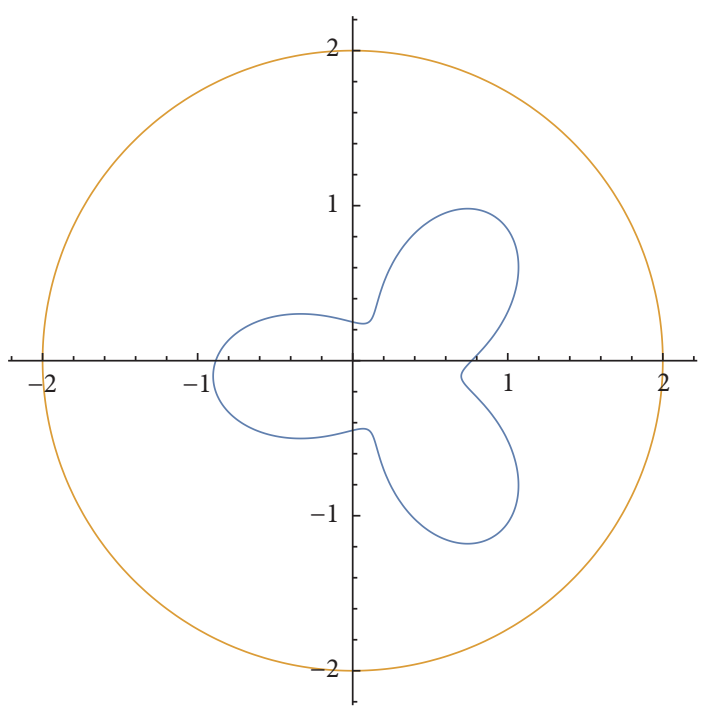

(a) Domain considered

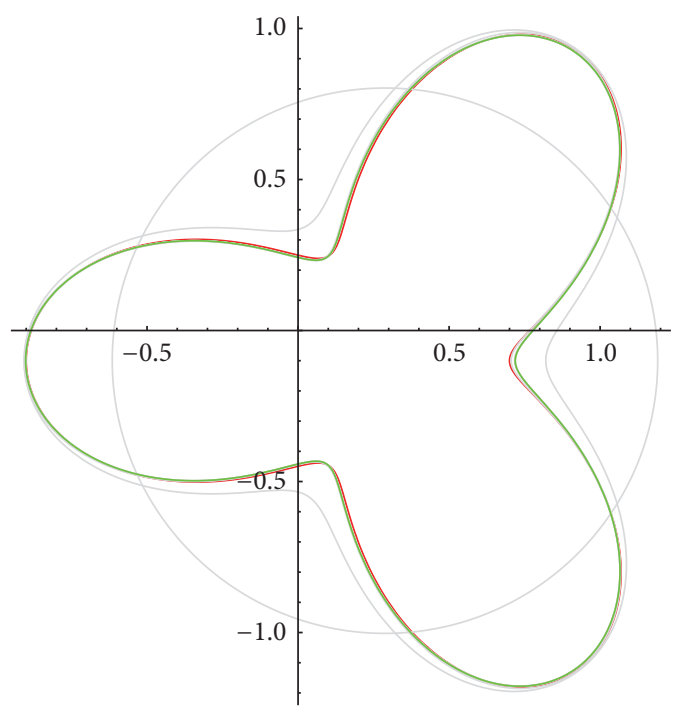

(b) Reconstruction, after 7 iterations, no noise

FIgURE 7: Case $\lambda>0$. Experiment with domain of radius 2.

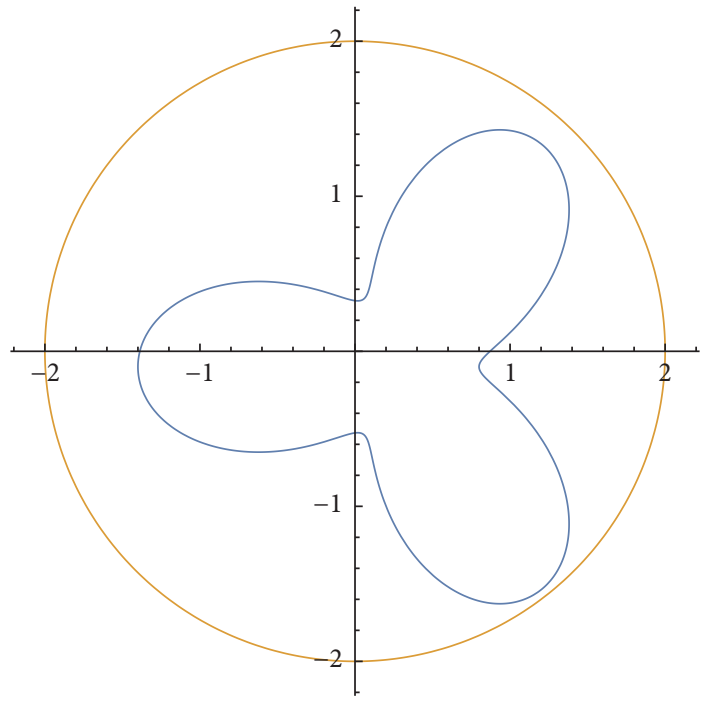

(a) Domain considered

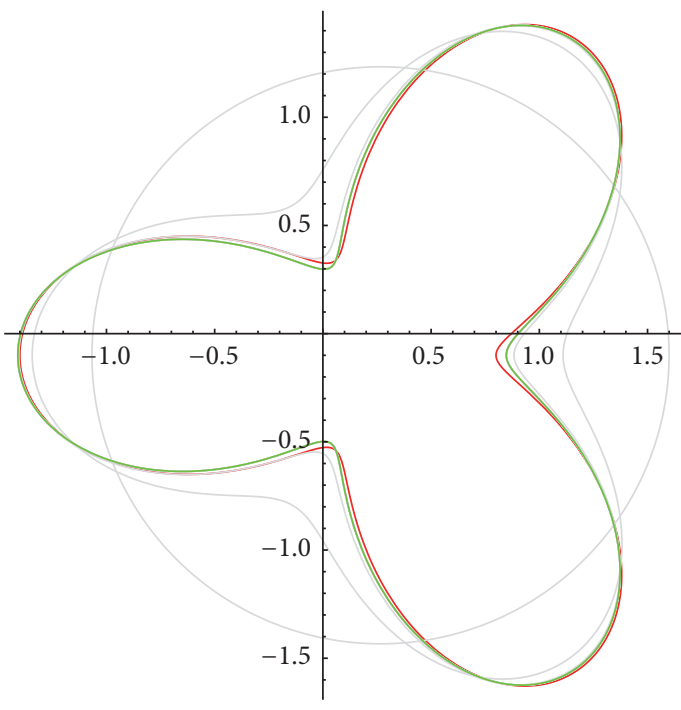

(b) Reconstruction, after 7 iterations, no noise

FIGURE 8: Case $\lambda>0$. Domain with radius 2. Boundary support close to boundary domain.

Figure 6(d). The reconstructions were again very efficient, even with considerable noise.

An experiment with the same kind of domain and source support was also implemented, but with different size. In Figure 8, the domain is a circle with radius 2, and the source support boundary is centered at $(0.3,-0.1)$ with parametrization $r(t)=0.8-0.4 \cos (3 t)$. The reconstruction, without noise, was very efficient (beyond the third iteration there is no visual difference), as shown in Figure 7(b).

Another experiment was done considering a source support having the parametrization, given by $r(t)=1.1-$ $0.6 \cos (3 t)$, closer to the domain boundary, as can be seen in Figure 8(a). This proximity did not influence the reconstruction, as it can be seen in Figure 8(b) (without noise) or in Figure 9, with relative noise of 1\%, 5\%, 10\%, and even 50\%.

Example 6. In this experiment, the original source support, $\omega$, was considered as the same square centered in $\left(x_{c}, y_{c}\right)=$ $(0.4,-0.2)$, and the domain, $\Omega$, was considered the unitary circle, as shown in Figure 10(a), and the Dirichlet data was considered null.

Again $M=36$ was considered, but in this case we took $\mathrm{NP}=11$. After 10 iterations, with approximately 40 seconds each, the reconstruction obtained, without noise, is shown in Figure 10(b). 


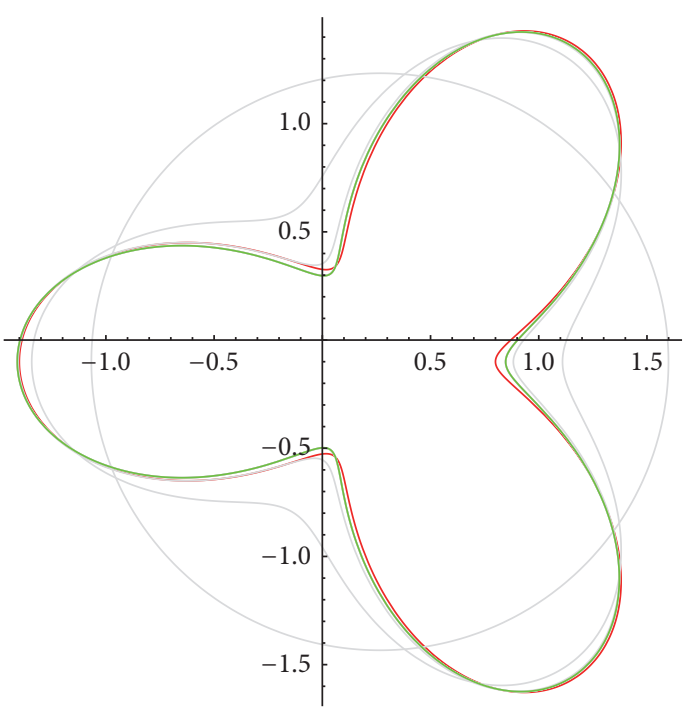

(a) $1 \%$

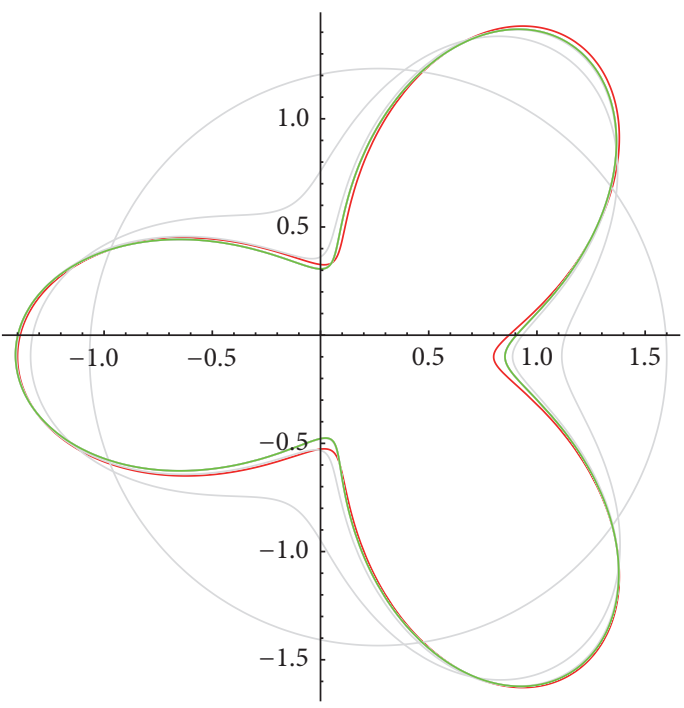

(c) $10 \%$

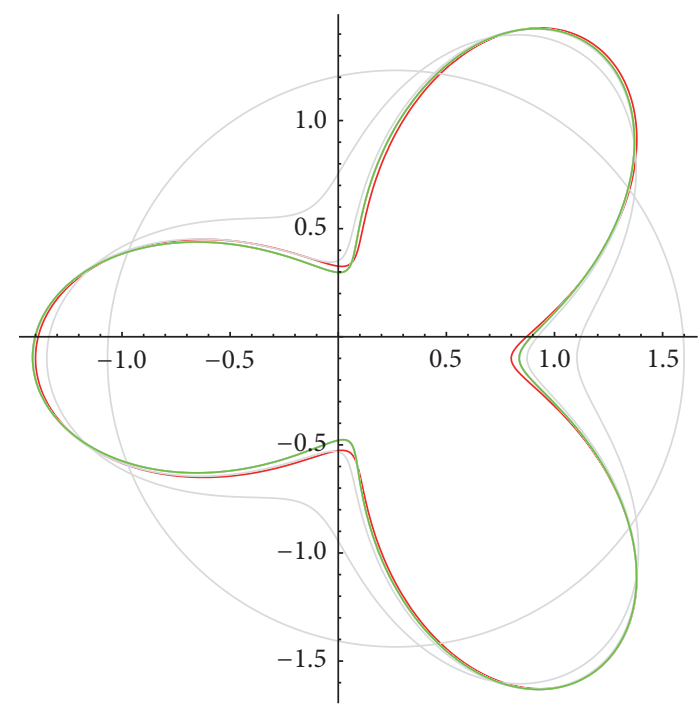

(b) $5 \%$

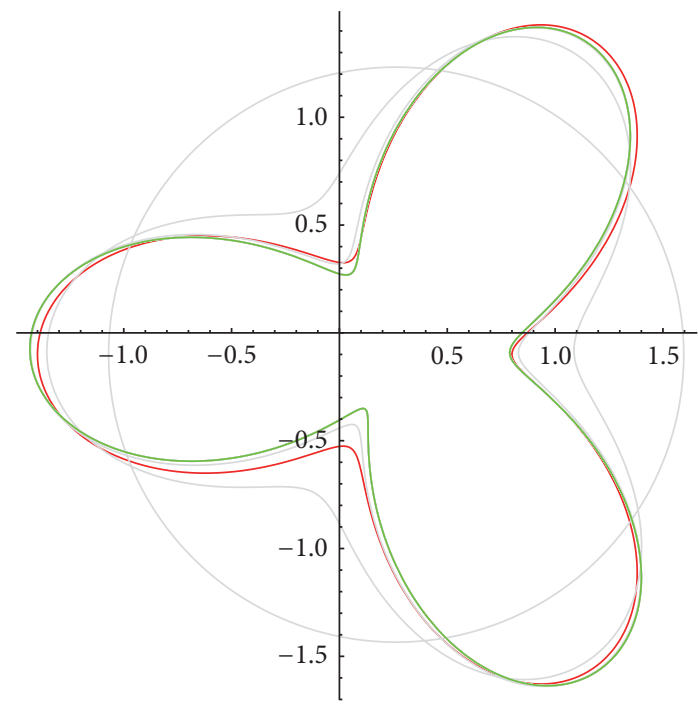

(d) $50 \%$

Figure 9: Case $\lambda>0$. Relative noise. Boundary support close to boundary domain.

4.2.4. Numerical Experiments for Boundary Reconstruction: Case $\lambda<0$. Now we considered $\lambda=-1$ and $\kappa:=\sqrt{\lambda}=$ $\sqrt{-1}=i$.

Example 7. In this experiment, the source support, $\omega$, was considered with center $\left(x_{c}, y_{c}\right)=(0.4,-0.2)$ and boundary parametrization $r(t)=0.3-0.15 \cos (3 t)$, inside an unitary circle domain $\Omega$, as shown in Figure 11(a), again with null Dirichlet boundary data.

The number of parameters in Fourier expansion was taken to be $\mathrm{NP}=7$. In the direct problem, 200 collocation points and 100 source points were taken for the MFS, and 160 collocation points and 80 source points were used in the MFS iterations.

The mean radius, calculated by (59), was $r_{M}=0.315357-$ $8.14923 \times 10^{-8} i$. So, we considered the initial parameter vector as $\left(\operatorname{Re}\left(r_{M}\right), 0,0,0,0,0,0\right)$, where $\operatorname{Re}\left(r_{M}\right)$ denotes the real part of $r_{M}$.

After 7 iterations, of approximately 65 seconds each, the reconstruction was obtained without noise shown in Figure 11(b), where the figure in red is the original and the figure in green is the reconstructed one. It can be observed that from the third iteration the results are very close.

Although the time in each iteration was larger, the reconstruction was better than with experiments with $\kappa$ real.

We had considered both kinds of noise in the Neumann measurements (relative noise of $1 \%, 10 \%$, and $30 \%$ and absolute noise of $1 \%$ and $5 \%$ ).

In experiments related to relative noise only 3 iterations were considered, due to the fast convergence. The results can be seen in Figure 12 (the figures in red are original and the figures in green are the reconstructed ones). 


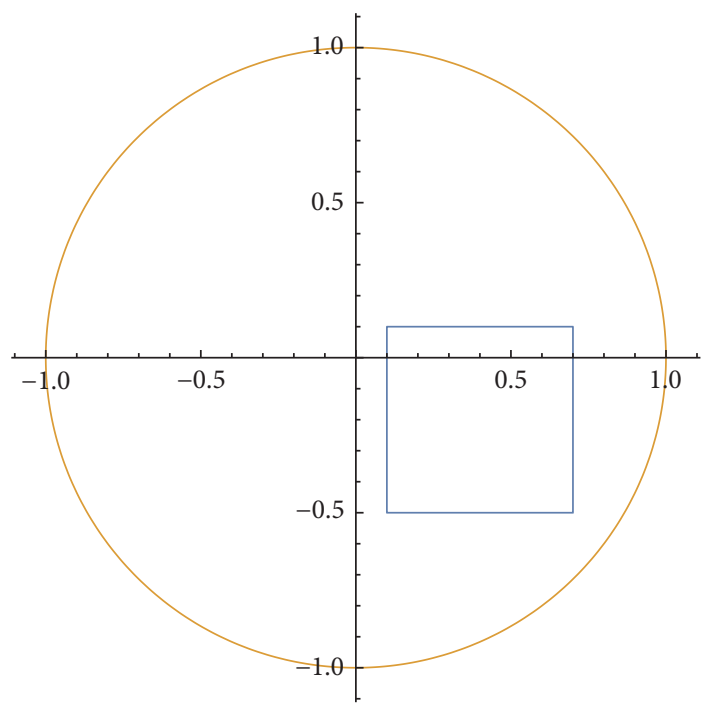

(a) Domain considered

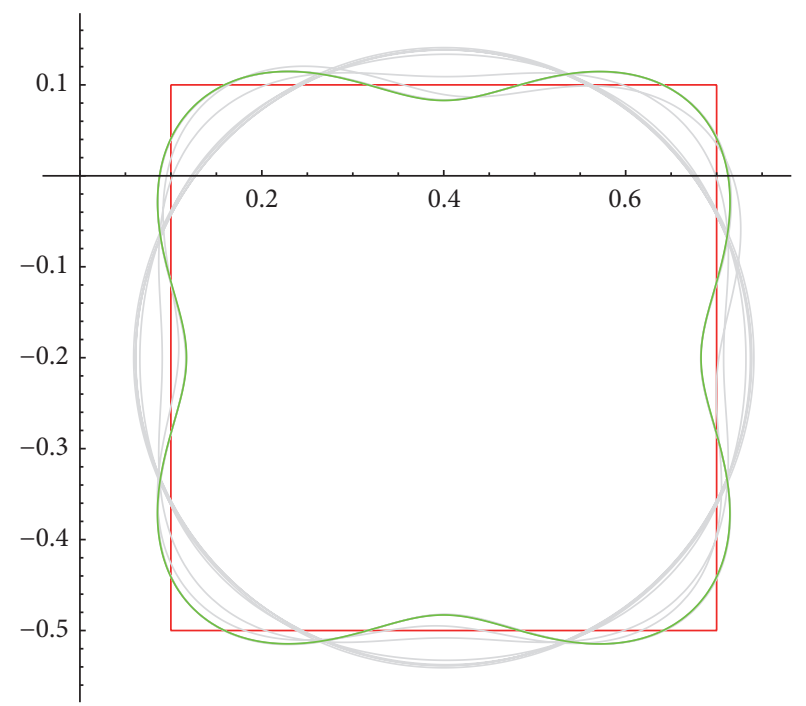

(b) Reconstruction, after 10 iterations, no noise

Figure 10: Case $\lambda>0$. Square support.

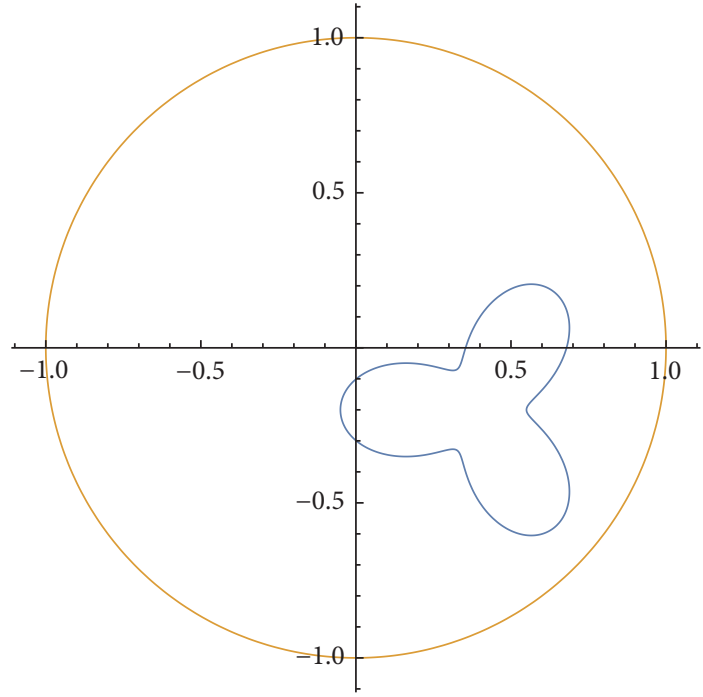

(a) Domain considered

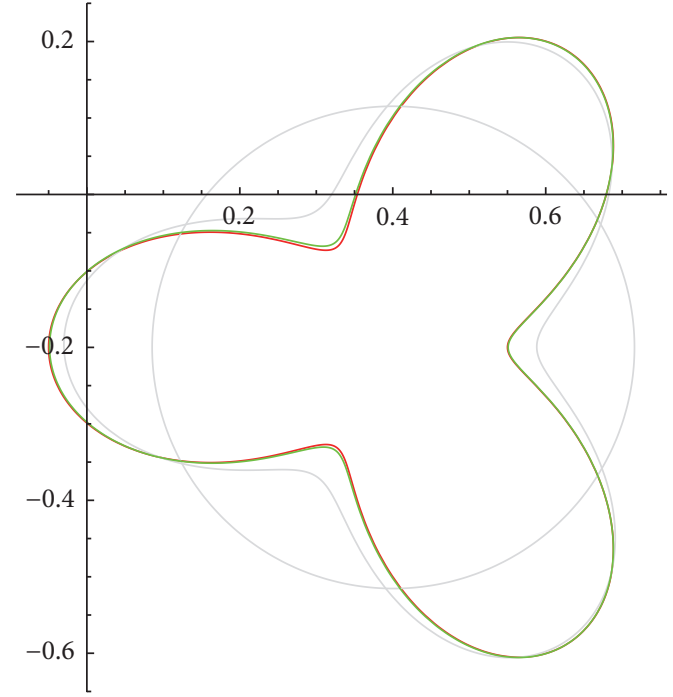

(b) Reconstruction, after 7 iterations, no noise

Figure 11: Case $\lambda<0$. Experiment with domain of radius 1.

In experiments with absolute noise, the method also converged quickly, after 3 iterations, but the reconstruction only maintained some meaning for a small amount of absolute noise (with 5\% the meaning was lost). The results can be seen in Figure 13.

Remark 14. Note that to avoid problems known as "inverse crimes," the MFS used for the simulation of the direct problem used different points from the MFS used for the inverse iterations. Furthermore, beside the addition of absolute and random noise, notice that in the square support example the boundary square shape could not be reproduced by a finite Fourier series expansion.

\section{Conclusions}

In this work, we studied the inverse characteristic source problem for Helmholtz equations. Firstly we proved that the associated direct problem is equivalent to a direct jump problem. This equivalence justifies the use of the Method of Fundamental Solution. Beside this, considering $\omega$ support of a characteristic source as a bounded symmetric star-shaped subset of the domain, we present a formula for the barycenter of $\omega$ and a shape reconstruction method. Numerical experiments related to barycenter determination and boundary reconstruction have shown very accurate results. 


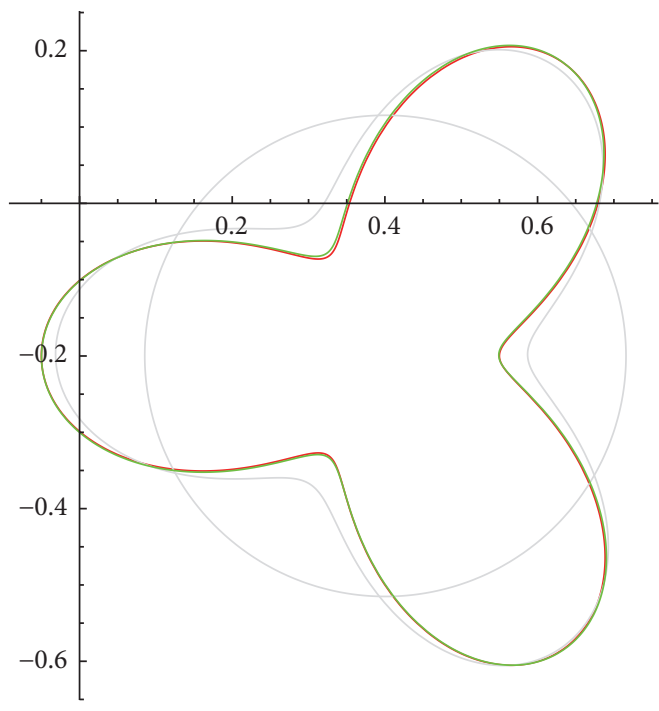

(a) $1 \%$

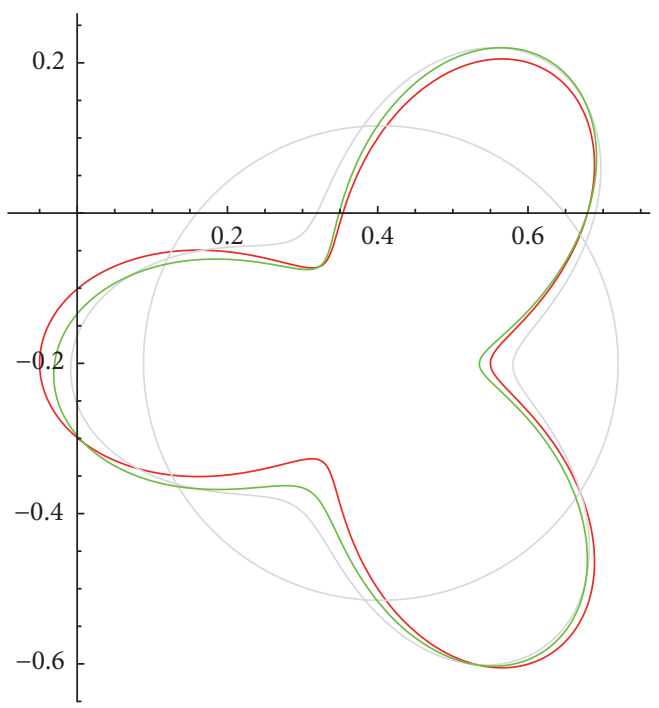

(b) $10 \%$

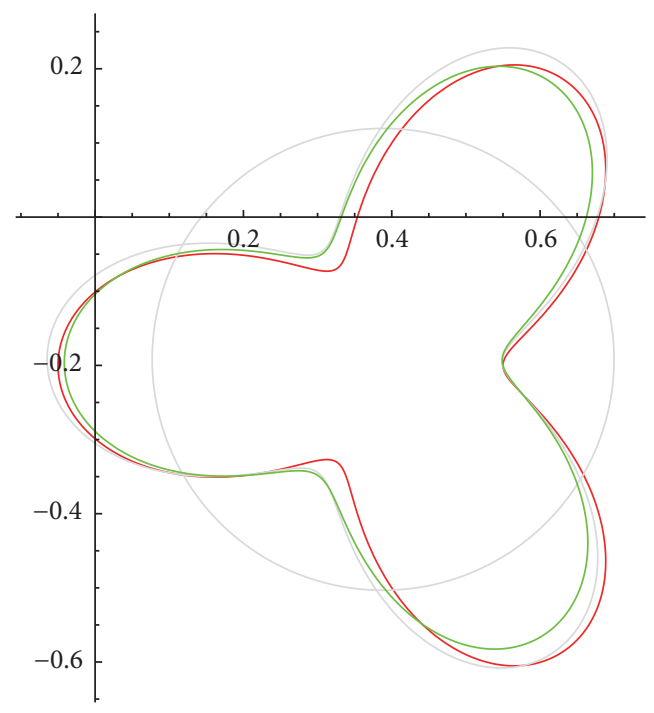

(c) $30 \%$

FIgURE 12: Case $\lambda<0$. Relative noise.

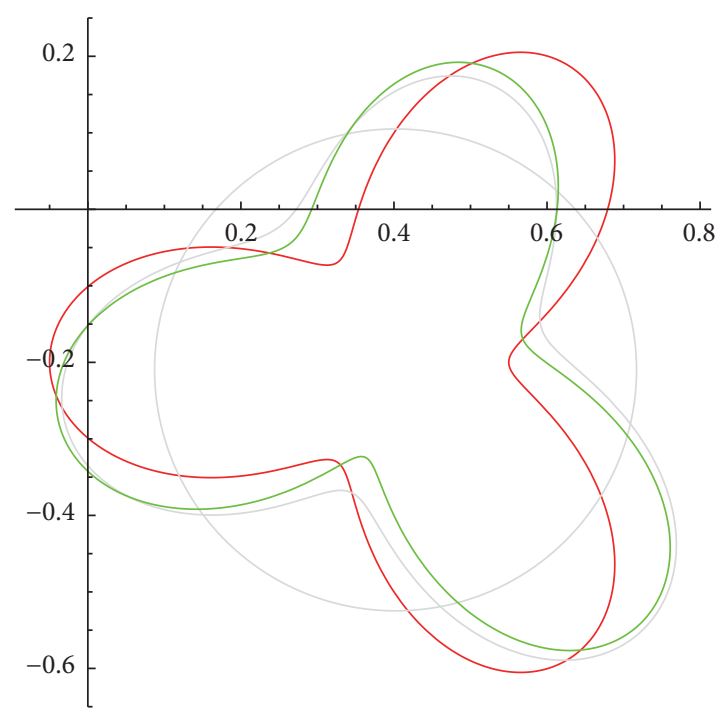

(a) $1 \%$

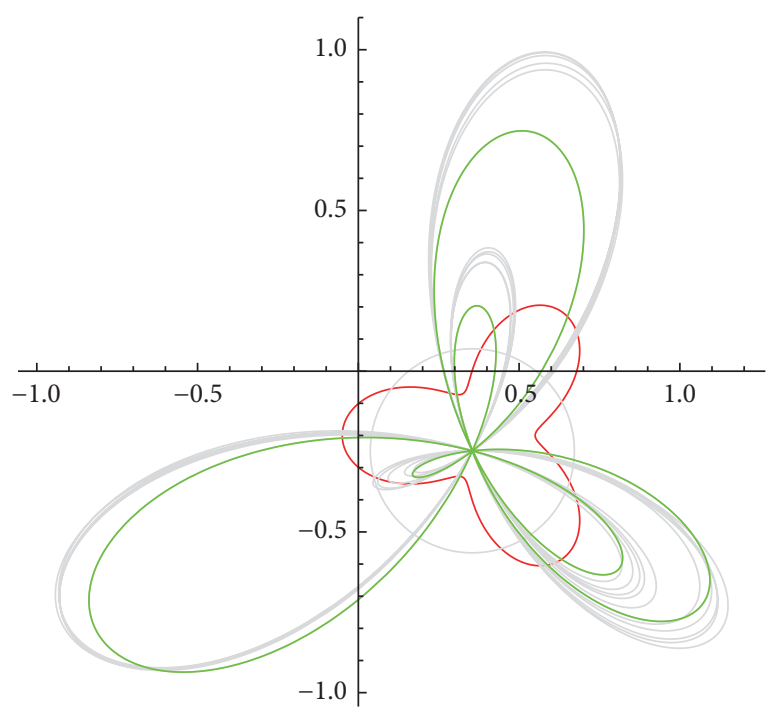

(b) $5 \%$

Figure 13: Case $\lambda<0$. Absolute noise. 


\section{Competing Interests}

The authors declare that there is no conflict of interests regarding the publication of this paper.

\section{Acknowledgments}

This work was partially financed by Brazilian agencies CNPq, Process no. 141829/2012-5, and CAPES, Process no. 99999.007941/2014-5.

\section{References}

[1] P. S. Novikov, "Sur le probléme inverse du potentiel," Doklady Akademii Nauk SSSR, vol. 18, pp. 165-168, 1938.

[2] M. Sakai, "A moment problem on Jordan domains," Proceedings of the American Mathematical Society, vol. 70, no. 1, pp. 35-38, 1978.

[3] V. Isakov, Inverse Source Problems, vol. 34 of Mathematical Surveys and Monographs, American Mathematical Society, Providence, RI, USA, 1990.

[4] A. El Badia and T. Ha-Duong, "An inverse source problem in potential analysis," Inverse Problems, vol. 16, no. 3, pp. 651-663, 2000.

[5] A. El Badia and T. Ha-Duong, "On an inverse source problem for the heat equation. Application to a pollution detection problem," Journal of Inverse and Ill-Posed Problems, vol. 10, no. 6, pp. 585-599, 2003.

[6] M. Andrle and A. El Badia, "On an inverse source problem for the heat equation. Application to a pollution detection problem, II," Inverse Problems in Science and Engineering, vol. 23, no. 3, pp. 389-412, 2015.

[7] C. J. S. Alves, M. J. Colaço, V. M. A. Leitão, N. F. M. Martins, H. R. B. Orlande, and N. C. Roberty, "Recovering the source term in a linear diffusion problem by the method of fundamental solutions," Inverse Problems in Science and Engineering, vol. 16, no. 8, pp. 1005-1021, 2008.

[8] B. Jin and L. Marin, "The method of fundamental solutions for inverse source problems associated with the steady-state heat conduction," International Journal for Numerical Methods in Engineering, vol. 69, no. 8, pp. 1570-1589, 2007.

[9] F. Hettlich and W. Rundell, "Iterative methods for the reconstruction of an inverse potential problem," Inverse Problems, vol. 12, no. 3, pp. 251-266, 1996.

[10] F. Hettlich and W. Rundell, "Recovery of the support of a source term in an elliptic differential equation," Inverse Problems, vol. 13, no. 4, pp. 959-976, 1997.

[11] N. C. Roberty and C. J. Alves, "On the identification of starshape sources from boundary measurements using a reciprocity functional," Inverse Problems in Science and Engineering, vol. 17, no. 2, pp. 187-202, 2009.

[12] W. Ring, "Identification of a core from boundary data," SIAM Journal on Applied Mathematics, vol. 55, no. 3, pp. 677-706, 1995.

[13] A. El Badia and T. Nara, "An inverse source problem for Helmholtz's equation from the Cauchy data with a single wave number," Inverse Problems, vol. 27, Article ID 105001, 2011.

[14] R. Kress and W. Rundell, "A nonlinear integral equation and an iterative algorithm for an inverse source problem," Journal of Integral Equations and Applications, vol. 27, no. 2, pp. 179-198, 2015.
[15] D. M. de Sousa and N. C. Roberty, "An inverse source problem for the stationary diffusion-advection-decay equation," Inverse Problems in Science and Engineering, vol. 20, no. 7, pp. 891-915, 2012.

[16] A. El Badia and T. Ha Duong, "Some remarks on the problem of source identification from boundary measurements," Inverse Problems. An International Journal on the Theory and Practice of Inverse Problems, Inverse Methods and Computerized Inversion of Data, vol. 14, no. 4, pp. 883-891, 1998.

[17] C. J. Alves, N. F. Martins, and N. C. Roberty, "Full identification of acoustic sources with multiple frequencies and boundary measurements," Inverse Problems and Imaging, vol. 3, no. 2, pp. 275-294, 2009.

[18] S. Acosta, S. Chow, J. Taylor, and V. Villamizar, "On the multifrequency inverse source problem in heterogeneous media," Inverse Problems, vol. 28, no. 7, 2012.

[19] A. Karageorghis, D. Lesnic, and L. Marin, "A survey of applications of the MFS to inverse problems," Inverse Problems in Science and Engineering, vol. 19, no. 3, pp. 309-336, 2011.

[20] L. C. Evans, Partial Differential Equations, vol. 19 of Graduate Studies in Mathematics, University of California, Berkeley, Calif, USA, 2nd edition, 2010.

[21] G. Fairweather and A. Karageorghis, "The method of fundamental solutions for elliptic boundary value problems," Advances in Computational Mathematics, vol. 9, article 69, 1998.

[22] G. Ala, G. Fasshauer, E. Francomano, S. Ganci, and M. McCourt, "The method of fundamental solutions in solving coupled boundary value problems for M/EEG," SIAM Journal on Scientific Computing, vol. 37, no. 4, pp. B570-B590, 2015.

[23] G. Ala, E. Francomano, G. E. Fasshauer, S. Ganci, and M. J. McCourt, "A meshfree solver for the MEG forward problem," IEEE Transactions on Magnetics, vol. 51, no. 3, 2015.

[24] C. J. Alves, "On the choice of source points in the method of fundamental solutions," Engineering Analysis with Boundary Elements, vol. 33, no. 12, pp. 1348-1361, 2009.

[25] A. H. Barnett and T. Betcke, "Stability and convergence of the method of fundamental solutions for Helmholtz problems on analytic domains," Journal of Computational Physics, vol. 227, no. 14, pp. 7003-7026, 2008.

[26] A. Karageorghis, D. Lesnic, and L. Marin, "A moving pseudoboundary method of fundamental solutions for void detection," Numerical Methods for Partial Differential Equations, vol. 29, no. 3, pp. 935-960, 2013.

[27] C. S. Chen, A. Karageorghis, and Y. Li, "On choosing the location of the sources in the MFS," Numerical Algorithms, vol. 72, no. 1, pp. 107-130, 2016. 


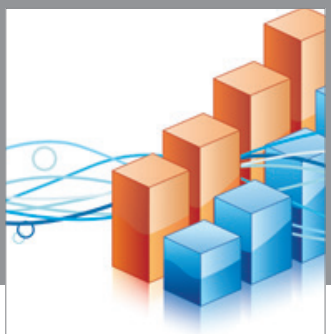

Advances in

Operations Research

vatem alat4

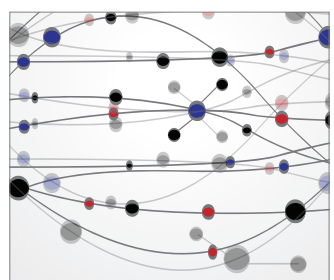

\section{The Scientific} World Journal
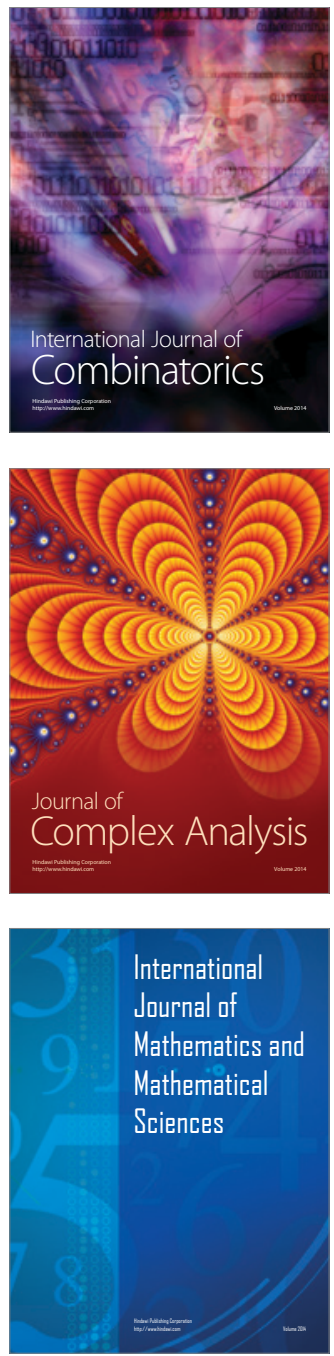
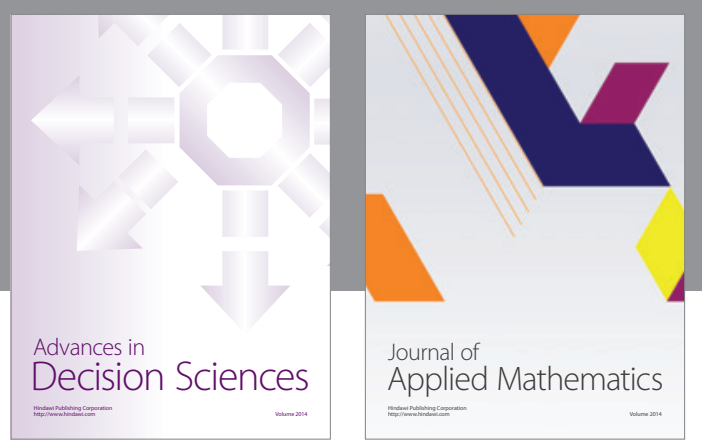

Algebra

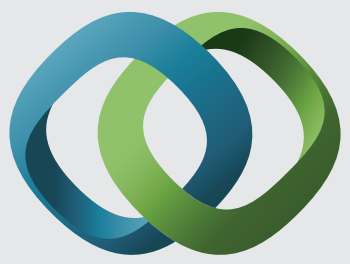

\section{Hindawi}

Submit your manuscripts at

https://www.hindawi.com
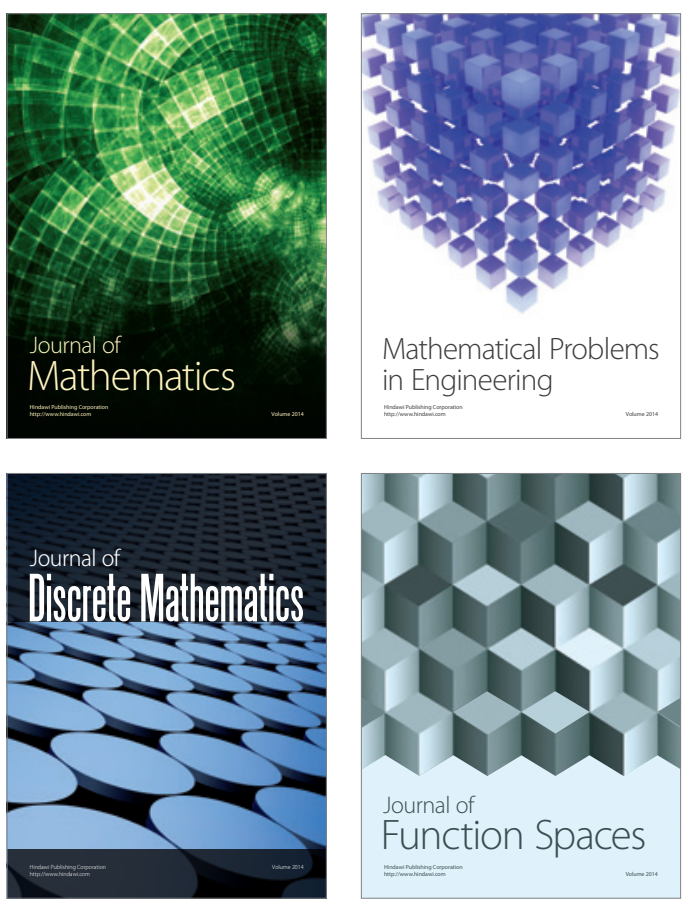

Mathematical Problems in Engineering
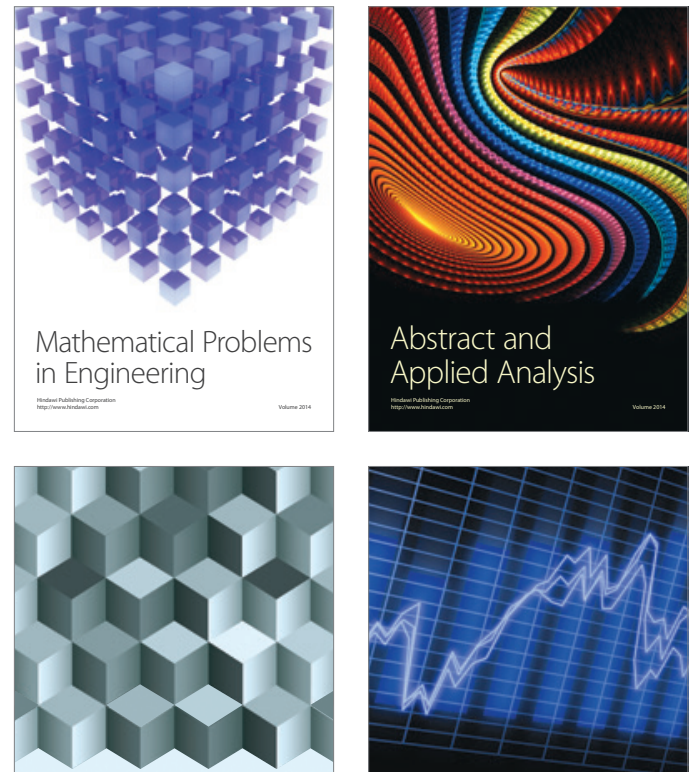

Journal of

Function Spaces

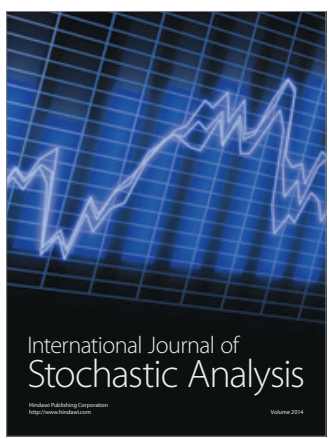

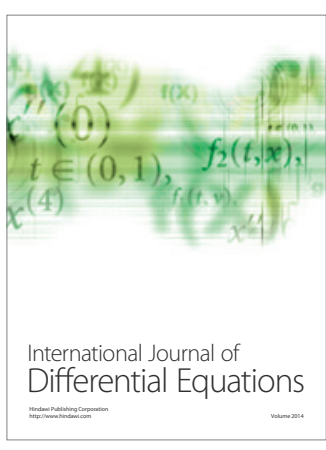
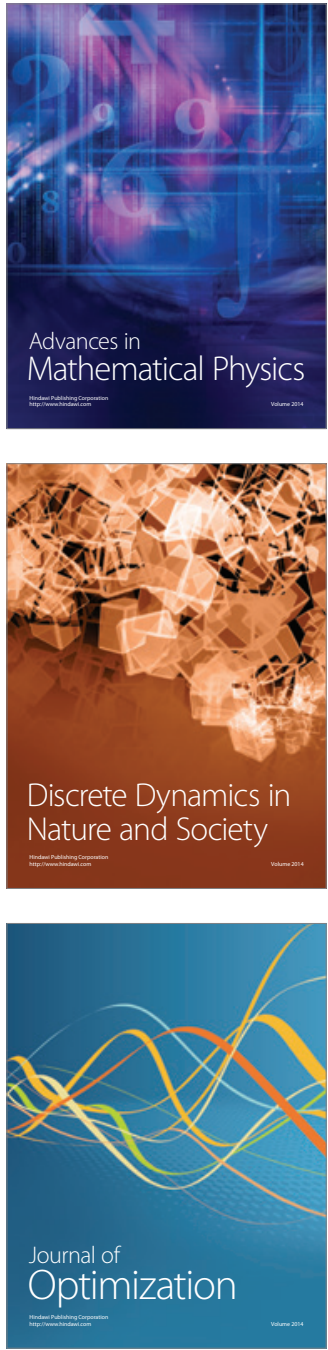